\title{
A STRONGER SYSTEM OF OBJECT THEORY AS A PROTOTYPE OF SET THEORY
}

\author{
KATUZI ONO
}

\section{Introduction}

We have introduced in our former work [1] a theory of mathematical objects which can be regarded as a prototype of set theory. We have been successful to imbed the Zermelo set-theory [3] without the axiom of choice in our system. However, it seems impossible to imbed the Fraenkel set-theory [4] in our system even without the axiom of choice. In this work, we introduce another system of object theory in which we can imbed the Fraenkel settheory without the axiom of choice. We shall denote our former system by $\mathbf{O Z}$ (object theory in the manner of the Zermelo set-theory) and the new system we are going to introduce in this work by OF (object theory in the manner of the Fraenkel set-theory). We shall also denote the Zermelo set-theory without the axiom of choice by $\mathbf{S Z}$ and the Fraenkel set-theory without the axiom of choice by SF.

As for description of our theory, we illustrate some notions and leading ideas properly important for $\mathbf{O F}$ in ordinary sentences to give vivid images of them. Concerning proofs, our reasoning can be formulated in the so-called classical predicate-logic of the first order, such as the logical system LK formulated by Gentzen [6]. As it is advisable to minimize careless mistakes in our real deductions by formulating our theory in a formal system, we have formulated our proofs in the way of describing formal deductions introduced in our former work [2]. In the following, we denote our logical system, the combination of the logic and our way of description, by PLK (practical system of logic equivalent to Gentzen's $\mathbf{L K}$ ). It should be also remarked here that our system assumes existence of some objects. In fact, any proposition of the form $\exists x(\mathfrak{I}(x) \rightarrow \mathfrak{A}(x))$ is provable in LK. For the most part of theorems and meta-theorems in this work, however, we indicate their logical basis only. We

Rẹceived September 21, 1962; Revised December 11, 1962. 
give exact formal proofs described in PLK only for theorems and meta-theorems properly important for $\mathbf{O F}$.

Originally, we do not need any special term-symbols such as $\varnothing,\{\varnothing\},\{x, y\}$, $f(x)$, etc. All the symbols denoting objects can be taken as variables. For convenience' sake, however, we introduce a special kind of term symbols in the set theory described in Section (15), because we can show that there is no danger of appearing new contradictions caused by introducing these term symbols. (We do not give further any detailed proof of it in this work.) Variables for obiects in general are denoted by capital Latin letters. In Section (11), however, we introduce set variables denoted by small Latin letters which are used especially for sets.

Just as the system $\mathbf{O Z}$, the new system $\mathbf{O F}$ is also an extremely simple system. It has only one primitive notion "membership" (notation " $\in$ ") and assumes only one axiom scheme. OF is a system closely related to SF in the sense that the only one axiom scheme of $\mathbf{O F}$ resembles the axiom of replacement of $\mathbf{S F}$, just as $\mathbf{O Z}$ is closely related to $\mathbf{S Z}$ in the sense that the only axiom scheme of $\mathbf{O Z}$ resembles the aussonderung axiom of $\mathbf{S Z}$. OF is a system stornger than $\mathbf{O Z}$ in the sense that we can imbed $\mathbf{O Z}$ in $\mathbf{O F}$ (See (14.6) ${ }^{1}$.), whereas it seems impossible to imbed OF in OZ. The main purpose of this work is to introduce the system $\mathbf{O F}$ and to prove further that a theory of sets equivalent to $\mathbf{S F}$ can be established in $\mathbf{O F}$ quite naturally.

Just as in $\mathbf{O Z}$, we describe the axiom scheme of $\mathbf{O F}$ by making use of the satellite notion. The satellite notion of $\mathbf{O F}$ is a modification of the satellite notion of OZ, but we must admit that the definition of the latter seems slightly simpler than that of the former. So, we can not say that $\mathbf{O F}$ is an improved system of $\mathbf{O Z}$. However, the imbedding process of $\mathbf{S Z}$ as well as of $\mathbf{S F}$ in $\mathbf{O F}$ is much simpler than that of SZ in OZ. Moreover, the Fraenkel set-theory looks like a better ground than the Zermelo set-theory to construct mathematical theories in it. So we believe that it is an important problem to seek for a theory as simple as possible in which we can construct the Fraenkel set-theory. Our new system $\mathbf{O F}$ is a trial system intended to be a basic system of this kind. In this work too, we discuss nothing concerning the axiom of choice,

1) In the numbering of the forms $(a)$ and $(a, b)$ in this work, $a$ and $b$ denote the numbers of a section and a paragraph respectively. 
but it may be possible to prove consistency of the axiom by following Gödel's proof, [5].

OF has the only one primitive notion "membership", naturally a binary relation denoted by " $\in$ ". In $\mathbf{O Z}$, we have succeded to establish a set theory equivalent to SZ by restricting our object field to producible objects (See $O Z$ (3.2.1) $)^{2)}$ and $O Z$-(5.2.1) together with $O Z \cdot(2.2 .1)$ and $O Z$-(3.1.1).) and at the same time by defining membership " $\in$ " in terms of proto-membership $\epsilon$, the only one primitive notion of OZ. (See $O Z$-(4.9.1) together with $O Z$-(4.6.1).) To imbed $\mathbf{S F}$ in $\mathbf{O F}$, we have also to restrict our object field to sets (defined in (11.1)), but it is not necessary to modify our primitive notion membership $\in$ any more. We can really develop a set theory in OF in a simpler way than in $\mathbf{O Z}$ because of this situation. Anyway, membership $\in$ of $\mathbf{O F}$ corresponds to proto-membership $\epsilon$ of $\mathbf{O Z}$.

In developing the two systems of our object theories $\mathbf{O Z}$ and $\mathbf{O F}$, essential difference lies in the notion of unit objects. In OZ, unit objects are defined in connection with identity $=$ of $\mathbf{O Z}$, while in $\mathbf{O F}$, unit objects are defined in connection with equality $=$ of $\mathbf{O F}$ which is defined as $X=Y \equiv \forall S(S \in X \equiv S \in Y)^{3)}$ and consequently which corresponds to proto-equality $\simeq$ of OZ. (See (2.1), $O Z$ (1.2.3), OZ-(1.2.5), and $O Z$-(1.4.3).) However. it should be noticed here also that these notions coincide if they are properly interpreted in set theories such as the system SF.

In $\mathbf{O F}$ too, the notion of satellites is essentially important. Also the satellite notion of $\mathbf{O F}$ coincides with the satellite notion of $\mathbf{O Z}$, if they are properly interpreted in SF. However, they show an apparent difference in development of our object theories by the difference between the unit-object notions of the two systems or, in other words, by the difference between identity of $\mathbf{O Z}$ and equality of $\mathbf{O F}$.

In describing $\mathbf{O F}$, we employ further two kinds of meta-symbols for denoting conditions and relations. First kind of such symbols are special symbols such as $=$ used for abbreviations of long expressions. Examples of notations

2) The numberings of the forms $O Z \cdot(a), O Z \cdot(a, b)$, and $O Z \cdot(a, b, c)$ denote $(a),(a, b)$, and $(a, b, c)$ of our former work [1], respectively.

3) Any formula of the form $\mathfrak{A} \equiv \mathfrak{B}$ is read " $\mathfrak{A}$ is defined by $\mathfrak{B}$ ", which means that the notation $\mathfrak{A}$ stands for the proposition $\mathfrak{B}$. 
expressed by symbols of this kind are $X=Y, X \subseteq Y, X \varnothing Y, X \sigma Y, \emptyset(X), \sigma(X)$, etc. $X \subseteq Y$ means $\forall S(S \in X \rightarrow S \in Y)$ and $\varnothing(X)$ means $\forall S \cdot S \notin X$ respectively. (See Definitions (2.15) and (6.1).) Symbols such as $\varnothing$ and $\sigma$ are used for denoting conditions on a single variable as well as for denoting binary relations. For example, $\varnothing(X)$ is expressed also as $X \varnothing Y$ when the condition $\varnothing(X)$ is regarded as a binary relation. (See Definition (6.1).) Usually, the condition and the binary relation denoted by a common symbol are closely related to each other. I.et us take up now another example $\sigma$. Just as in $\mathbf{O Z}$, we define also in $\mathbf{O F}$ the condition $\sigma(P)$ at first and define the relation $X \sigma Y$ thereafter by making use of the notation $o(P)$. (See $O Z-(1.1 .3),(1.4)$, and (1.5.).) The conditions and relations denoted by these special symbols can be of course defined in terms of membership $\in$, the only primitive notion of OF.

Another kind of meta-symbols are used for denoting forms of formulas. For this purpose, we use capital German letters together with capital Greek letters which are used especially for binary relations. For example,

$$
\exists P \forall X(X \in P \equiv \cdot \mathfrak{A}(X) \wedge X \in M)^{4)}
$$

is a proposition scheme corresponding to the aussonderung axiom, and

(B) $\forall X Y Z^{5)}(X \Gamma Z \wedge Y \Gamma Z \cdot \rightarrow X=Y) \rightarrow \exists P \forall X(X \in P \equiv \exists Z(X I Z \wedge Z \in M))$

is a propoistion scheme corresponding to the axiom of replacement.

As we express the forms of propositions mostly by symbols for binary relations of this kind, it looks convenient to introduce here some combinations and operations of binary relations.

Together with the relation products of binary relations (See $O Z-(1.1 .1)$ ), we use the logical combinations of the forms $I \diamond \Delta$ of $I$ and $\Delta$, which are defined by $X(\Gamma \diamond \Delta) Y$ ₹ $X I Y \diamond X \Delta Y$, where $\diamond$ stands for any one of $\wedge, \vee$, $\rightarrow$, and $\equiv$. Similarly, we can define the negation $\neg T$ and the inverse relation $\Gamma\left(\right.$ or $\Gamma^{-1}$ ) of $\Gamma$ in a natural way. Notations such as $\notin, \neq, \ni, \supseteq$, etc. can be also used if adequate. By the way, we use also expressions such as

4) In describing axioms and theorems, we usually omit universal quantifiers of a formula standing at its top and having the whole formula as their scope.

5) Quantifiers of the forms $\forall X \cdots Z$ and $\exists X \cdots Z$ naturally stand for $\forall X \cdots \forall Z$ and $\exists X \cdots \exists Z$ respectively. 
$X \Gamma Y \Delta Z, X \Gamma Y \Delta Z \Theta W$, etc. in case of need, which naturally stand for $X \Gamma Y \wedge Y \Delta Z$, $X \Gamma Y \wedge Y \Delta Z \wedge Z \Theta W$, etc., respectively. Naturally, the relation product is associative in the sense that any relation of the form $(\Gamma \Delta) \Theta$ is equivalent ${ }^{6}{ }^{1}$ to $\Gamma(\Delta \Theta)$. Accordingly, we usually omit parentheses denoting the order of multiplications. Moreover, the relation product is monotone in the sense that the relation $\Gamma \Delta$ implies $^{\text {i) }}$ the relation $\Theta \Lambda$ if $\Gamma$ and $\Delta$ imply $\Theta$ and $A$ respectively. So, any relation of the form $\Gamma(\Delta \wedge \Theta)$ implies $\Gamma \Delta$ as well as $\Gamma \Theta$, and any relation of the from $(\Gamma \wedge \Delta) \Theta$ implies $\Gamma \Theta$ as well as $\Delta \Theta$. Also, the relation product is distributive over the combination $\vee$ in the sense that any relation of the form $\Gamma(\Delta \vee \Theta)$ is equivalent to $\Gamma \Delta \vee \Gamma \Theta$ and any relation of the form $(\Gamma \vee \Delta) \Theta$ is equivalent to $\Gamma \Theta \vee \Delta \Theta$.

Further, we define the comprehension operator $\{\Gamma$ ) by

$$
X\{\Gamma) Y \text { ₹ } \forall S(S \in X \equiv S \Gamma Y),
$$

where $S$ is a variable which does not occur in $I$. We denote the inverse relation of $\{\Gamma)$ naturally by $(\Gamma\}$. We have introduced the operaor " $\{$ )" already in our former work [2], although we did not give it the name "comprehension operator" there (See $\left.P D-(3)^{8)}\right)$. This operation makes our description amazingly simple. For example, equality $=$ of $\mathbf{O F}$ is denoted simply by $\{\in)$ and the unit-object relation is denoted simply by $\{=)$. Namely, $X\{=) Y$ means that $X$ is a unit object of $Y$. (See (2.26).)

Concerning equality in $\mathbf{O F}$, we can not expect that any relation of the form $=I$ as well as any relation of the form $\Gamma=$ is equivalent to $\Gamma$. Any relation $\Gamma$ which is equivalent to $=\Gamma$ (or to $\Gamma=$ ) is called left invariant (or right invariant). If a relation is left invariant as well as right invariant, we call it invariant. (See Definition (2.2).)

Although the satellite notion of $\mathbf{O F}$ is really somewhat different from the satellite notion of $\mathbf{O Z}$, the interpreted satellite-notions of these two systems coincide in the system SF, so we have no need to give further explanation for the purpose that one should have a vague image of the satellite notion of $\mathbf{O F}$.

\footnotetext{
6) Any two binary relations $\Phi$ and $\Theta$ are called equivalent if and only if $\forall X Y \cdot X(\Phi \equiv \Theta) Y$ holds.

7) We say that the binary relation $I$ implies the binary relation $\Delta$ if and only if $\forall X Y \cdot X(\Gamma \rightarrow \Delta) Y$ is provable.

8) We refer to Section (a) of our former work [2] by $P D-(a)$.
} 
(Detailed illustration is given in (1.7).) Here we would like to show a feature of our system OF from a rather imaginative side.

The axiom scheme of $\mathrm{OZ}$ is a form of formulas obtained by replacing a membership relation of the aussonderung axiom by the satellite relation of $\mathbf{O z}$. There is a good reason to expect that one may obtain another nice generating principle by replacing a membership relation of the axiom of replacement by a suitably defined satellite-relation. Because the axiom of replacement is somewhat stronger than the aussonderung axiom, one may expect that an object theory stronger than $\mathrm{OZ}$ can be established on the basis of this generating principle regarding it as a sole assumption of the theory. Compared with the aussonderung axiom or with the axiom scheme of $\mathbf{O Z}$, however, the usual expression of the axiom of replacement seems too complicated, having the assumption of uniqueness of the mapping. For example, compared with the formula (A), the feature of (B) looks too complicated, because it has the uniqueness assumption $\forall X Y Z(X I Z \wedge Y \Gamma Z \cdot \rightarrow X=Y)$.

By virtue of the comprehension operator, we know a very extensive type of unique mappings having a simple form. Namely, we can easily show that any relation of the form $\{I$ ) defines a unique mapping (regarding $Y\{T) X$ as a mapping $X$ to $Y$ ) with respect to equality $=$ i.e. $\{\in$ ). (See (2.7).) Although it seems impossible to express every unique mapping $\Delta$ in the form $\{\Gamma$ ), we can express any left invariant unique mapping $\Delta$ in the form $\{T)$. Because, in SF, every relation can be regarded as invariant, we need not afraid that this restriction may cause any essential difficulty. By the way, how can we express every left invariant unique relation $\Delta$ in the form $\left\{I^{\prime}\right)$ ? If there is an object $Z$ satisfying $Z \Delta X, Y \Delta X$ is equivalent to $Y\{\in \Delta) X$. However, if there is no such $Z$, we can make use of the well-known Russell paradox. Namely, let us define $T$ by

$$
U \Gamma V ₹ \cdot U \in \Delta V \vee(\neg \exists W \cdot W \Delta V: \wedge U \notin U) \text {. }
$$

Then, we can prove that $\Delta$ is equivalent to the relation $\left\{I^{\circ}\right)$. An exact proof of this fact is given in (2.31) at the end of Section (2). In this connection, it should be particularly noticed that the whole theory of Sections (2) and (3) can be developed without assuming any axiom. (Compare $P D$-(3).)

In fact, the formula 
(E)

$$
\exists P \cdot P\{\{\Gamma) \in) M \quad \text { i.e. } \quad \exists P \forall X(X \in P \equiv: \exists Z \cdot X\{\Gamma) Z \in M)
$$

is a special case of the proposition scheme (B), the proposition scheme corresponding to the axiom of replacement, but it is a proposition scheme of so extensive character that we are apt to regard it as a substitute of the axiom of replacement. Attracted by the extremely simple form of the formula (E), we have attempted to define the satellite relation $\sigma$ in such a way that the modified formula

$$
\exists P \cdot P\{\{\Gamma \mid \sigma) M
$$

of (E) will be a sole axiom scheme of an object theory called OF which can be taken as a prototype of the set theory SF.

We have been successful to do this only by modifying the satellite notion of $\mathrm{OZ}$ in accordance with the modification of the unit-object notion. The modification of the unit-object notion seems indispensable to our plan, because we can regard all the binary relations of the form $\{\Gamma$ ) as unique mappings only since we take the relation $\{\in$ ) as equality. (See (2.7).) In accordance with this modification, we define at first $X \subseteq Y$ by $\forall S(S\{\in) \in X \rightarrow S\{\in) \in Y$ ) as a modification of the relation $X \subseteq Y$ which literally corresponds to the relation $X \subseteq Y$ of $\mathbf{O Z}$. We define next $\sigma(P)$ by $\forall S(S \in \in P \equiv S \subseteq \in P)$. Then, we define $X \sigma Y$ by $\forall P(\sigma(P) \wedge Y \in \in P \text {. } \rightarrow X \in \in P)^{9)}$. (See (1.2), (1.3), (1.4), (1.5), and (2.15).) Anyway, the interpreted satellite-notions of $\mathbf{O Z}$ and of $\mathbf{O F}$ coincide in SF. As pointed out in $O Z-(7)$, we can prove in SF that there is a set formed by all the interpreted satellites of any set. This leads to the result that $\mathbf{O F}$ is consistent if $\mathbf{S F}$ is so. Our new system $\mathbf{O F}$ is formally introduced in Section (1), and a short sketch of a consistency proof of $\mathbf{O F}$ relative to $\mathrm{SF}$ is given in (1.7).

Sections (2)-(15) are devoted to show that we can really establish the object theory $\mathrm{OF}$ on the basis of the only one axiom scheme (F) which is strong enough to establish a set theory equivalent to SF (so, according to $O Z$ (7) and $O Z-(6)$, also an object theory equivalent to $O Z$ and a set theory equivalent to SZ) in it. (See especially (14.6) and (15.11).) Only in Section (10), we take a somewhat long way round by developing a theory of natural

9) In $O Z$, we can define $X \sigma Y$ also as $\forall P(\sigma(P) \wedge Y \in \in P: \rightarrow X \in \in P)$. 
numbers in our object theory OF. To take a shorter course, we have only to give an example of infinite sets. Anyway, it is really a long series of troublesome work to develop the object theory of starting from the sole axiom scheme (F).

As our primitive notion $\in$ seems only right-invariant (See (2.5). It seems impossible to prove that $\in$ is left-invariant.), we can expect only for a special kind of objects $X$ that $X$ contains as its members every object which is equal to a member of $X$. Such objects $X$ are called normal (formally defined in (2.27)). One can easily see that the axiom of extensionality is equivalent to the assertion that all the sets are normal. It should be particularly remarked here that every object generated by an axiom of the form $(F)$ is normal. (We can prove this by (2.8), (2.10), and (2 29).)

In $\mathbf{O F}$ too, we define regularity. However, regularity of $\mathbf{O F}$ is slightly different from that of Oz. To define regularity, we use the notion of descendents. We call any object $X$ satisfying a condition of the form $X=\in \cdots \in Y$ (the number of $\in$ 's is $0,1,2, \ldots$ ) a descendent of $Y$. (The descendent relation is formally defined in (7.1). See also (7.7).) By making use of the descendent notion, we define regularity of $\mathbf{O F}$ also in such a way that, for any regular object $X$, there may be no infinite descending chain $X \ni X_{1} \ni X_{2} \ni \cdots$. (Compare (8.1) and OZ-(2.2.1). See also (8.2), illustration.) Any object whose descendents are all normal is called totally normal (formally defined in (9.1)). We can establish the theory of OF exclusively in the field of regular and totally normal objects which we simply call sets. (See (11.1).) The main result of this work is that the set theory SF is nothing but an object theory established exclusively in the field of sets. (See especially Section (15). Compare Gandy [7]. )

The relation between $\mathbf{O F}$ and $\mathbf{S F}$ resembles to the relation between the theory of natural numbers without complete induction and the theory of natural numbers with complete induction. (We denotes these two systems hereafter by $\mathbf{N}_{0}$ and $\mathbf{N}$ respectively.) $\mathbf{O F}$ and $\mathbf{S F}$ have the common generating principle of the form ( $\mathrm{F}$ ) just as $\mathbf{N}_{0}$ and $\mathbf{N}$ have the common generating principle that every number is followed by another number. Possibly there may be many systems $\mathbf{O F}$ of object theory having (F) as their sole generating principle, just as that there may possibly be many systems $\mathbf{N}_{0}$ of natural- 
number theory having the same generating principle that every number is followed by another number. The ordinary natural-number system $\mathbf{N}$ can be regarded as the minimum system of these systems of natural numbers. Similarly, the ordinary set theory SF looks like the minimum system of the object theories having the same generating principle $(F)$.

The complete induction of $\mathbf{N}$ positively asserts that $\mathbf{N}$ is really the minimum system among those systems $\mathbf{N}_{0}$. The system $\mathbf{S F}$, however, has no principle literally corresponding to the complete induction. In fact, it seems impossible to confirm by axioms of SF that the system SF is really the minimum system among those systems which have the same generating principles as that of $\mathbf{S F}$. So, it might be possible that a sufficiently small system of object theory having certain generating principles can be regarded as a system of set theory by suitable interpretation. This was the case for $\mathbf{O Z}$, because a sufficiently small system of objects, the system of producible objects, can be regarded as SZ if we define membership and equality suitably for the system. In $\mathbf{O F}$ too, we can construct a theory equivalent to SF by restricting the field of objects to the field of sets, i.e. the field of regular and totally normal objects. (See Definitions (8.1), (9.1), and (11.1). See also Section (15).) However, regularity and total normality can never be proved to be characteristic of objects belonging to the minimal system among those systems OF having ( $F$ ) as their sole generating principle.

By the axiom scheme (F), we can prove existence of suitably defined unit objects, sum objects, and power objects of any object (See (5.3), (5.2), and (5.4).), and also existence of suitably defined pair objects of any two objects (See (6.11) and also Definition (6.9).). However, it is extremely remarkable that we can prove these theorems even when we replace our satellite relation by the literal interpretation of the satellite relation (denoted here by $\underline{\sigma}$ ) of $\mathbf{O Z}$. Namely, $\underline{\sigma}$ is the relation defined by

$$
X \underline{\sigma} Y ₹ \forall P(\underline{\sigma}(P) \wedge Y \in \in P . \rightarrow X \in \in P)^{10)},
$$

where $\underline{\sigma}(P)$ stands for $\forall S(S \in \in P \equiv S \subseteq \in P)$.

Now, for convenience' sake, let us denote by $\mathbf{O H}$ the system of object theory having

\footnotetext{
10) See the foot-note 9 ).
} 
(H)

$$
\exists P \cdot P(\{\Gamma) \underline{\sigma}) M
$$

as its sole axiom scheme. The satellite notion of $\mathbf{O H}$ is surely simpler than that of $\mathbf{O F}$. Moreover, we can prove existence of unit objects, sum objects, power objects of any object, and also existence of pair objects of any two objects in the system $\mathbf{O H}$. We can further show in $\mathbf{O H}$ that membership $\in$ implies the relation $\underline{\sigma}$, so the example proof of $P D-(3)$ is also valid for $\mathbf{O H}$. Hence, in $\mathbf{O H}$ too, there exists no object containing all the objects as its members. Because any object $R$ defined by $R\{\{\in) \underline{\sigma}) M$ (proved to be existing by the axiom scheme of $\mathbf{O H}$ ) contains all the objects $X$ satisfying $X \underline{\sigma} M$ (we can prove reflexivity of $\{\in$ ) without any assumption), $X \sigma M$ can not hold for all objects $X$. Hence, $\underline{\sigma}(P)$ must hold for some $P$. Since reflexivity of the relation $\subseteq$ can be proved also without any assumption, there is surely a nonvacant object $P$ in the system $\mathbf{O H}$ which satisfies $\forall S(S \in P \rightarrow S \in \in P)$. At the first glance, one might suppose that this object $P$ is an example of infinite objects, but in reality, we have never been able to confirm by the axiom scheme (H) only that $P$ contains infinitely many members. We can prove in our system OF that there is surely an object formed by all the natural numbers (defined in (10.4)) which is an example of infinite objects in all respects, so, our modification of the satellite notion has been effective for our purpose anyway. (See (10.5), (10.7), and (10.15).)

\section{(1) The system OF}

In this Section, we introduce the system OF formally at first $((1.1)-(1.6))$, and next we give an illustration for the system.

(1.1) The binary relation $\in$ (membership) is the only primitive notion of the system $\mathbf{O F}$.

(1.2) DF. $X \in Y \mp X\{\in) \in Y$. The binary relation $\hat{\epsilon}$ is called the generalized membership. Any object $X$ satisfying $X \hat{\in} Y$ is called a generalized member of $Y$.

(1.3) DF. $X \subseteq Y ₹ \forall S(S \subseteq X \rightarrow S \in Y)$. The binary relation $\subseteq$ is called the generalized inclusion. Any object $X$ satisfying $X \subseteq Y$ is called a generalized subobject of $Y$.

(1.4) DF. $\sigma(P) \rightleftharpoons \forall \forall(S \in \in P \equiv S \subseteq \subseteq \in P)$. 
(1.5) DF. $X \sigma Y \rightleftharpoons \forall P(\sigma(P) \wedge Y \in \in P \cdot \rightarrow X \in \in P)$. The binary relation $\sigma$ is called the satellite relation. Any object $X$ satisfying $X \sigma Y$ is called a satellite of $Y$.

(1.6) Axiom scheme. All the formulas of the form

$$
\exists P \cdot P\left\{\{\Gamma)_{\sigma}\right) M
$$

and formulas of this form only are axioms of our system OF, where any number of free variables other than $P$ may occur in the binary relation $\Gamma$.

(1.7) Illustration. $\exists P \cdot P\left\{\{\Gamma)_{\sigma}\right) M$ can be also expressed as

$$
\exists P \forall X\left(X \in P \equiv X\{\Gamma)_{\sigma} M\right)
$$

and $X\{\Gamma)_{\sigma} M$ can be expressed as $\exists Y\left(\forall S(S \in X \equiv S \Gamma Y) \wedge Y_{\sigma} M\right)$. Accordingly, $X\{\Gamma)_{\sigma} M$ means that $X$ is an object formed by all the objects bearing the relation $\Gamma$ with a satellite $Y$ of $M$, and $P\left\{(\Gamma)_{\sigma}\right) M$ means that $P$ is an object formed by all the objects $X$ satisfying the condition $X\{\Gamma)_{\sigma M}$. In OZ, the satellite notion is introduced in such a way, that any proto-member as well as any subobject as well as any unit object of an object is a satellite of the object, and that the satellite relation is reflexive and transitive. (See $O Z$-(1.2.11), $O Z$-(1.2.12), OZ-(1.4.9), OZ-(1.2.13), and OZ-(1.2.14).) Each axiom of $\mathbf{O Z}$ asserts that all the satellites of an object satisfying a certain condition form a new object. (See $O Z$-(1.1.4).) We wish to introduce the satellite notion of OF similarly to the satellite notion of $\mathbf{O Z}$ but at the same time in a form suitably modified for the axiom scheme of $\mathbf{O F}$.

It is not necessary in $\mathbf{O F}$ to draw any distinction between proto-membership and membership. According to the axiom scheme of $\mathbf{O F}$, any member of an object generated by an axiom is characterized by their members, so the protoequality of $\mathbf{O Z}$ looks like a better example for defining equality in OF rather than identity of OZ. Just replacing " $\epsilon$ " in the definition of proto-equality of $\mathbf{O Z}$ by " $\in$ ", we define equality $=$ of $\mathbf{O F}$. Thus equality notion of $\mathbf{O F}$ deviates from identity of $\mathbf{O Z}$ in some extent. (Compare Definition (2.1) with $O Z$-(1.2.5) and $O Z$-(1.2.3).) In accordance with this deviation, the notion of unit objects has to be slightly changed. Moreover, in defining satellites, inclusion has to be replaced by a new notion, the generalized inclusion $\subseteq$ which is a little weaker than inclusion. According to Definitions (1.4) and (1.5), in OF too, 
membership $\in$, the generalized inclusion $\subseteq$, and the unit-object relation $\{=$ ) (defined in (2.26)) implies the satellite relation $\sigma$. Also, the satellite relation $\sigma$ is reflexive and transitive. (See (3.2), (3.3), (3.4.), and (3.1).)

If we interpret "objects" of $\mathbf{O F}$ as "sets" of SF and " $\in$ " of $\mathbf{O F}$ as " $\in$ " of SF, we can easily see that "巳”" of OF is interpreted as " $\subseteq$ " of SF and the satellite relation $\sigma$ of $\mathbf{O F}$ is interpreted as the relation " $\hat{\jmath}$ ", which is defined by

$$
x \hat{\sigma} y ₹ \forall p(\hat{\sigma}(p) \wedge y \in \in p \cdot \rightarrow x \in \in p)^{11)},
$$

where $\hat{\sigma}(p)$ is a condition on $p$ defined by $\hat{\sigma}(p) ₹ \forall s(s \in \in p \equiv s \subseteq \in p)$. Namely, the relation $\hat{\sigma}$ of $\mathbf{S F}$ is the same relation " $\hat{\sigma}$ " that is defined in $O Z-(7)$.

In SF holds that, for any set $m$, there is a set $n$ formed by all the satellites (in the interpreted sense) of $m$. (This is shown in $O Z$-(7).) For any binary relation $\Gamma$ in $\mathrm{SF}$, the relation $x\{T) y$ (defined naturally as $\forall s(s \in x \equiv s \Gamma y)$ ) is evidently a unique mapping $y$ to $x$, because SF has the axiom of extensionality. As $p\{\{\Gamma) \hat{\sigma}) m$ expresses $\forall x(x \in p \equiv: \exists y \cdot x\{\Gamma) y \hat{\sigma} m)$ and further $\forall y(y \in n \equiv y \hat{\sigma} m)$ holds, so $\exists p \cdot p\{\{\Gamma) \hat{\sigma}) m$ can be also expressed as $\exists p \forall x(x \in p \equiv: \exists y \cdot x\{\Gamma) y \in n)$. This is a special case of the axiom of replacement of $\mathrm{SF}$, because $x\{\Gamma) y$ is a unique mapping $y$ to $x$. Accordingly, the interpreted proposition of every axiom of $\mathbf{O F}$ is provable in SF.

Thus the system $\mathrm{OF}$ can be imbedded in SF, so our object theory $\mathrm{OF}$ is consistent as far as the Fraenkel set-theory is so.

\section{(2) Equality and inclusion}

In this Section, we define two binary relations, equality $=$ and inclusion $\subseteq$, and also the notion normality. We describe also some elementary properties of these notions in connection with membership $\in$, the generalized membership $\hat{\epsilon}$, and the generalized inclusion $\subseteq$.

(2.1) DF. The relation $\{\in)$ is denoted by $=$ and is called equality.

(2.2) DF. Any binary relation is called right (or left) invariant if and only if $\Gamma=($ or $=\Gamma$ ) is equivalent to the relation $\Gamma$. If $\Gamma$ is right invariant as well as left invariant, it is simply called invariant.

11) See the foot-note 9 ). 
(2.3) Equality = is reflexive, symmetric, and transitive. Also, equality is invariant. (Proof. ${ }^{12 \prime}$ By (2.1).)

(2.4) Any binary relation $\Gamma$ is right (or left) invariant if the relation $\Gamma=$ (or $=\Gamma$ ) implies $\Gamma$. Consequently, $\Gamma$ is invariant if the relation $=\Gamma=$ implies Г. (Proof. By (2.2) and (2.:).)

(2.5) The membership $\in$ is right invariant. (Proof. By (2.1) and (2.4).)

(2.6) The membership $\in$ implies the generalized membership $\hat{\epsilon}$. (Proof. By (1.2), (2.1), and (2.3).)

(2.7) Any relation of the form $\{\Gamma)(\Gamma\}$ implies equality $=$. In other words, any relation of the form $\{\Gamma)$ is unique with respect to equality =, regarding $X\{\Gamma) Y$ as a mapping $Y$ to $X$. (Proof. By (2.1).)

(2.8) Any binary relation of the form $\Gamma \Delta$ is right invariant if $\Delta$ is so, and it is left invariant if $\Gamma$ is so. Consequently, the relation $\Gamma \Delta$ is invariant if $\Gamma$ is left invariant and $\Delta$ is right invariant. (Proof. By (2.2) and (2.4).)

(2.9) If two binary relations $\Gamma$ and $\Delta$ are both right (or left) invariant, the binary relations $\Gamma \wedge \Delta$ and $\Gamma \vee \Delta$ are both right (or left) invariant. (Proof. By (2.2) and (2.4).)

(2.10) Any relation of the from $\{\Gamma$ ) is left invariant. (Proof. By (1.2), (2.1), and (2.4).)

(2.11) For any right invariant relation, $\Gamma$ the binaty relation $\{\Gamma$ ) is invariant. (Proof. By (2.2), (2.3), (2.4), and (2.10).)

(2.12) The generalized membership $\hat{E}$ is invariant. (Proof. By (1.2), $(2.5),(2.8)$, and (2.10).)

(2.13) The generalized inclusion $\subseteq$ is reflexive and transitive. (Proof. By (1.3).)

(2.14) The relation $\hat{E} \subseteq$ is equivalent to the generalized membership $\hat{E}$. (Proof. By (1.3) and (2.13).)

(2.15) DF. $X \subseteq Y ₹ \forall S(S \in X \rightarrow S \in Y)$. The binary relation $\subseteq$ is called inclusion. Any object $X$ satisfying $X \subseteq Y$ is called a subobject of $Y$.

12) In any proof of this type, we indicate its logical basis only. 
(2.16) The inclusion $\subseteq$ is reflexive and transitive. (Proof. By (2.15).)

(2.17) Equality $=$ is equivalent to the relation $\subseteq \Lambda \supseteq$, where $\supseteq$ is the inverse relation of inclusion $\subseteq$. (Proof. By (2.1) and (2.15).)

(2.18) The inclusion $\subseteq$ is invariant. (Proof. By (2.4), (2.16), and (2.17).)

(2.19) The relation $\in \subseteq$ is equivalent to the membership $\in$. (Proof. By (2.15) and (2.16).)

(2.20) $X \subseteq Y \rightarrow \forall S(S \in X \rightarrow S \in Y$ ). (Proof. By (2.6) and (2.19).)

(2.21) $X \subseteq Y \equiv \forall S(S \in X \rightarrow S \in Y$ ). (Proof. By (1.2), (1.3), (2.1), (2.2), (2.6), (2.12), and (2.14).)

(2.22) The inclusion $\subseteq$ implies the generalized inclusion $\subseteq$. (Proof. By (2.20) and (2.21).)

(2.23) The generalized inclusion $\subseteq$ is invariant. (Proof. By (2.4), (2.13), (2.17), and (2.22).)

(2.24) The relation $\{\hat{E})$ implies the generalized inclusion $\subseteq$ (Proof. By (2.21).

(2.25) The inverse relation of $\{\subseteq$ ) i.e. the relation $(\widehat{\in}\}$ implies inclusion $\subseteq$. (Proof. By (2.6) and (2.15).)

(2.26) $X\{=) Y \rightarrow Y \in X$. Namely, the inverse relation of $\{=$ ) i.e. the relation $(=\}$ implies membership $\in$. (Any object $X$ satisfying $X\{=) Y$ is called a unit object of $Y$. The relation $\{=)$ is called the unit-object relation. Proof. By (2.3).)

(2.27) DF. Any object $X$ satisfying $X(€) X$ is called normal.

(2.28) Any object $X$ is normal if $\forall S(S € X \rightarrow S \in X)$. (Proof. By (2.6) and (2.27).)

(2.29) $X\{\Gamma) Y \rightarrow X\{€) X$ holds for any left invariant binary relation $\Gamma$. (Proof. By (1.2), (2.1), (2.2), (2.27), and (2.28).)

(2.30) The relation $\{€ \in$ ) is transitive. (Proof. By (2.12) and (2.29).)

(2.31) Any left invariant relation $\Delta$ can be expressed in the form $\{\Gamma$ ), if $\Delta \breve{\Delta}$ implies equality $=$, in other words, if the relation $\Delta$ is unique. 
$\operatorname{Proof}^{13)} / A \rightarrow c, d$.

A) Assume that $\Delta$ is left invariant and that $\Delta \breve{\Delta}$ implies $=$.

B) Define $\Gamma$ by $U \Gamma V$ ₹ $U \in \Delta V \vee(\neg \exists W \cdot W \Delta V: \wedge U \notin U)$.

c)) $\Delta$ implies $\{\Gamma) \quad / c A \rightarrow c d . \quad$ cA) $\forall X Y !^{14)} X \Delta Y$.

cb)) $\forall S(S \in X \rightarrow S \Gamma Y) \quad / c b A \rightarrow c b c . \quad$ cbA) $\quad \forall S ! S \in X$.

cbb) $S \in \triangle Y \quad / c b A, c A$. cbc) $S \Gamma Y \quad / c b b, B$.

cc) $\quad \forall S(S \Gamma Y \rightarrow S \in X) \quad / c c A \rightarrow c c g . \quad$ ccA) $\forall S ! S \Gamma Y$.

ccb) $S \in \Delta Y \vee(\neg \exists W \cdot W \Delta Y: \wedge S \notin S) \quad / c c A, B$.

cec) $\exists W \cdot W \Delta Y \quad / \mathbf{A}$.

ced) $S \in \Delta Y \quad / c c b, c c c . \quad$ cce) $\exists Z ! S \in Z \Delta Y \quad / c c d$.

cef) $Z=X \quad / c A, c c e, A$.

ccg) $S \in X \quad / c c e, c c f,(2.5),(2.2)$.

cd) $X\{\Gamma) Y / c b, c c$.

d) $\quad\{\Gamma)$ implies $\Delta \quad / d A \rightarrow d h . \quad$ dA) $\quad \forall X Y ! X\{\Gamma) Y$.

db) $\quad \exists W \cdot W \Delta Y \quad / d b A \rightarrow d b e$ (reductio ad absurdum).

dbA) Assume $\neg \exists W \cdot W \Delta Y$.

dbb) ) $\forall S(S \in X \rightarrow S \notin S) \quad / d b b A \rightarrow d b b e . \quad$ dbbA) $\quad \forall S ! S \in X$.

dbbb) $S \Gamma Y \quad / d b b A, d A$.

dbbc) $S \in \Delta Y \vee(\neg \exists W \cdot W \Delta Y: \wedge S \notin S) \quad / d b b b, B$.

dbbd) $\quad>S \in \Delta Y \quad / d b A . \quad$ dbbe) $S \notin S \quad / d b b c, d b b d$.

dbc) $\quad \forall S(S \notin S \rightarrow S \in X) \quad / d b c A \rightarrow d b c d . \quad$ dbcA) $\quad \forall S ! S \notin S$.

dbcb) $>\exists W \cdot W \Delta Y: \wedge S \notin S \quad / d b A, d b c A$ d dbce) $\quad S \Gamma Y / d b c b, B$.

dbed) $S \in X \quad / d b c c, d A$.

dbd) $\forall S(S \in X \equiv S \notin S) \quad / d b b, d b c$.

dbe) contradiction / $d b d$ (the Russell paradox).

de) $\exists T ! T \Delta Y / d b$.

dd) $\quad \forall S(S \in X \rightarrow S \in T) \quad / d d A \rightarrow d d g . \quad$ ddA) $\quad \forall S ! S \in X$.

ddb) $S \Gamma Y / d d A, d A$.

dde) $S \in \Delta Y \vee(\neg \exists W \cdot W \Delta Y: \wedge S \notin S) \quad / d d b, B$.

ddd) $S \in \Delta Y \quad / d d c, d b$.

13) Any proof of this type is a formal proof described in PLK.

14) Nominating quantifiers (See $P D$-(1).) of the forms $\forall X \cdots Z$ ! and $\exists \mathrm{X} \cdots Z$ ! naturally stand for $\forall X \cdots \forall Z$ ! and $\exists X \cdots \exists Z$ ! respectively. 


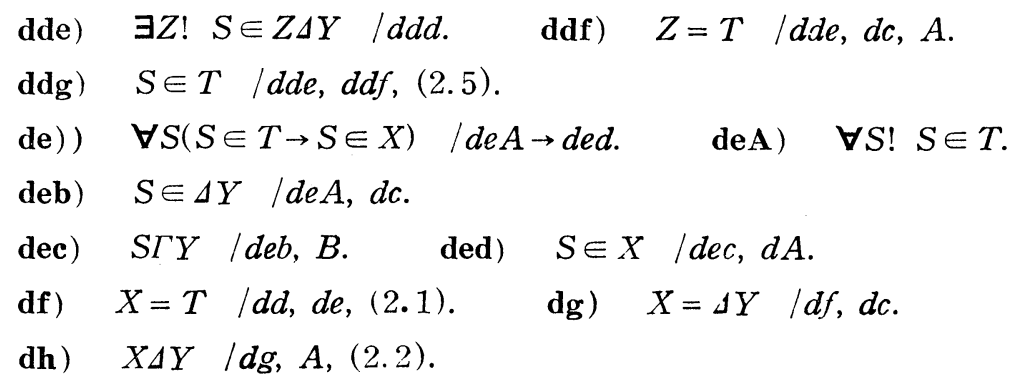

(3) Fundamental properties of the satellite relation $\sigma$.

(3.1) The satellite relation $\sigma$ is reflexive and transitive. (Compare OZ(1.2.13) and $O Z$-(1.2.14). Proof. By (1.5).)

(3.2) The membership $\in$ implies the satellite relation $\sigma$.

Proof $/ A \rightarrow b . \quad$ A) $\quad \forall X Y ! X \in Y$.
b)) $X \sigma Y \quad / b A \rightarrow b l,(1.5)$.
bA) $\forall P ! \sigma(P) \wedge Y \in \in P$.
bb) $Y \subseteq \in P \quad / b A,(1.4)$.
bc) $X \in \widehat{\subseteq} \in P \quad / A, b b$.
bd) $X \hat{\in} \in P \quad / b c,(2.21)$.
be) $X=\in \in P \quad / b d,(1.2),(2.1)$.
bf) $\exists Z ! X=Z \in \in P \quad / b e$.
bg) $Z \subseteq \in P \quad / b f, b A,(1.4)$.
bh ) $X \subseteq Z \quad / b f,(2.17)$.
bi) $X \subseteq Z \quad / b h,(2.22)$.
bj) $\quad X \subseteq \subseteq \in P \quad / b i, b g . \quad$ bk) $\quad X \subseteq \in P \quad / b j,(2.13)$.
bl) $X \in \in P \quad / b k, b A,(1.4)$

(3.3) The generalized inclusion $\subseteq$ implies the satellite relation $\sigma$. (Compare $O Z$-(1.2.12). Proof. By (1.4), (1.5), and (2.13).)

(3.4) The unit-object relation $\{=)$ implies the satellite relation $\sigma$.
Proof $/ A \rightarrow b$.
A) $\forall X Y ! X\{=) Y$.

b)) $X \sigma Y \quad / b A \rightarrow b e,(1.5)$.

bA ) $\quad \forall P ! \sigma(P) \wedge Y \in \in P$.

bb) $\exists Z ! \quad Y \in Z \in P \quad / b A$.

bc) $\quad X \subseteq Z \quad / b c A \rightarrow b c d,(2.21) . \quad$ bcA) $\quad \forall S ! S \in X$.

bcb) $S=Y \quad / A, b c A . \quad$ bcc) $\quad S=\in Z \quad / b c b, b b$.

bed) $S \in Z \quad / b c c,(1.2),(2.1)$.

bd) $X \subseteq \in P \quad / b c, b b . \quad$ be) $\quad X \in \in P \quad / b A, b d,(1.4)$.

(3.5) The satellite relation $\sigma$ is invariant. (Proof. By (2.4), (2.17), (2.22), (3.1), and (3.3).) 


\section{(4) Generating principles}

In connection with the axiom scheme of $\mathbf{O F}$, some generating principles, including those corresponding to the axiom scheme of $\mathbf{O Z}$ or to the aussonderung principle of $\mathbf{O Z}$, hold in OF. (See $O Z$-(1.3.1).) As they seem very powerful for the later exposition, we describe them in this Section.

(4.1) $\neg \forall T \cdot T \in M$. (There is no object formed by all the objects.)

This theorem is really important. However, we can prove this theorem by a slight modification of the example proof given in $P D$-(3), employing (2.1), (2.2), (2.3), (2.5), (3.2), and the axiom scheme (1.6). (See the foot-notes 4) and 5) of $P D$ i.e. [2].)

(4.2) $\exists P \cdot P\{=\Gamma \wedge \sigma) M$. (This is a meta-theorem of OF corresponding to the axiom scheme of $\mathrm{OZ}$. Propositions of the form $\exists P \cdot P\{\Gamma \wedge \sigma) M$ in general seem unprovable in OF. Even when we take up this proposition scheme, we can not regard it as a literal interpretation of the axiom scheme of $\mathbf{O Z}$, because the definition of the satellite relation $\sigma$ of $\mathbf{O F}$ is not a literal interpretation of the satellite relation of OZ. (Compare OZ-(1.1.4). Compare also (1.2), (1.3), and (1.4) with $O Z$-(1.1.2) and $O Z$-(1.1.3).)

$$
\begin{aligned}
& \text { Proof } / \mathrm{A} \rightarrow i . \quad \text { A) Define } \Delta \text { by } X \Delta Y ₹ \cdot Y \Gamma M \rightarrow X \in Y \text {. } \\
& \text { b) } \exists P ! P\left\{\{\Delta)_{\sigma}\right) M \text { /axiom. c) } \forall T\left(T \in P \equiv T\{\Delta)_{\sigma} M\right) \quad / b \text {. } \\
& \text { d) } \forall T(T \in P \equiv \exists Z(\forall U(U \in T \equiv U \Delta Z) \wedge Z \sigma M)) \quad / c \text {. } \\
& \text { e) } \forall T\left(T \in P \equiv \exists Z\left(\forall U(U \in T \equiv \cdot Z \Gamma M \rightarrow U \in Z) \wedge Z_{\sigma} M\right)\right) \quad / d, A \text {. } \\
& \text { f)) } \forall T(T \in P \rightarrow T(=\Gamma \wedge \sigma) M) \quad / f A \rightarrow f i . \quad \text { fA }) \quad \forall T ! T \in P \text {. } \\
& \text { fb) } \exists Z ! \forall U(U \in T \equiv \cdot Z \Gamma M \rightarrow U \in Z) \wedge Z \sigma M \quad l e, f A \text {. } \\
& \text { fc)) } Z \Gamma M / f c \mathrm{~A} \rightarrow f c c \text { (reductio ad absurdum). f(cA) Assume } \neg Z \Gamma M \text {. } \\
& \text { fcb) } \forall U \cdot U \in T \quad / f b, f c A \text {. fcc) contradiction } / f c b,(4.1) \\
& \text { fd) } \forall U(U \in T \equiv U \in Z) \quad / f b, f c . \quad \text { fe) } \quad T=Z \quad / f d,(2.1) \text {. } \\
& \text { ff) } T=\Gamma M \quad / f e, f c . \quad \text { fg) } \quad T_{\sigma} Z \quad / f e,(2.17),(2.22),(3.3) \text {. } \\
& \text { fh) } \quad T \sigma M / f g, f b,(3.1) . \quad \text { fi) } \quad T(=\Gamma \wedge \sigma) M \quad / f f, f h \text {. } \\
& \text { g) ) } \quad \forall T(T(=\Gamma \wedge \sigma) M \rightarrow T \in P) \quad / g A \rightarrow g h . \quad \text { gA) } \quad \forall T ! T=\Gamma M \wedge T \sigma M . \\
& \text { gb) } \exists S ! T=S \Gamma M \quad / g A \text {. } \\
& \text { ge)) } \forall U(U \in T \equiv \cdot S \Gamma M \rightarrow U \in S) \quad / g c a, g c b \text {. } \\
& \text { gea) } \forall U(U \in T \equiv U \in S) \quad / g b,(2.1) \text {. } \\
& \text { gcb) } \forall U(U \in S \equiv \cdot S \Gamma M \rightarrow U \in S) \quad / g b \text {. }
\end{aligned}
$$


gd) $\quad S_{\sigma} T \quad / g b,(2.17),(2.22),(3.3) . \quad$ ge) $\quad S_{\sigma} M \quad / g d, g A,(3.1)$.

gf) $\forall U(U \in T \equiv \cdot S \Gamma M \rightarrow U \in S) \wedge S \sigma M \quad / g c$, ge.

gg) $\exists Z \forall U(U \in T \equiv \cdot Z \Gamma M \rightarrow U \in Z) \wedge Z \sigma M) \quad / g f$.

gh) $T \in P \quad / e, g g$.

h) $\forall T(T \in P \equiv T(=\Gamma \wedge \sigma) M) \quad / f, g . \quad$ i) $\quad P\{=\Gamma \wedge \sigma) M \quad / h$.

(4.3) $\exists P \cdot P\{=\Gamma \wedge \subseteq) M . \quad$ (Compare $O Z$-(1.3.1). Proof ${ }^{15}$ We can take an object, say $P$, satisfying $P\{=(\Gamma \wedge \subseteq) \wedge \sigma) M$ by (4.2). $\quad P\{=\Gamma \wedge \subseteq) M$ can be proved by $(2.2),(2.3),(2.23)$, and (3.3).)

(4.4) $\exists P \cdot P\{=(\Gamma \wedge \in)) M$. (A meta-theorem of OF corresponding to the aussonderung axiom of SZ. Compare $O Z$-(1.3.1). Proof. We can take an object, say $P$, satisfying $P\{=(\Gamma \wedge \in) \wedge \sigma) M$ by (4.2). $P\{=(\Gamma \wedge \in)) M$ can be proved by (2.17), (2.22), (3.1), (3.2), and (3.3).)

(4.5) $\exists P \cdot P(\{\Gamma) \in) M$. (A meta-theorem of OF corresponding to the axiom of replacement of $\mathbf{S F}$.)

Proof $/ A \rightarrow d . \quad$ A) Define $\Delta$ by $X \Delta Y \equiv X\{\Gamma) \in M$.

b) $\exists Q ! Q\left\{\{\Gamma)_{\sigma}\right) M$ /axiom. c) $\exists P ! P\{=(\Delta \wedge \in)) Q \quad /(4.4)$.

d)) $P\{\{\Gamma) \in) M \quad / d a, d b$.

da) ) $\quad \forall X(X \in P \rightarrow X\{\Gamma) \in M) \quad / d a A \rightarrow d a d . \quad$ daA $) \quad \forall X ! X \in P$.

dab) $X=\Delta Q \quad / d a A, c . \quad$ dac $) \quad X=\{\Gamma) \in M \quad / d a b, A$.

dad) $X\{\Gamma) \in M \quad / d a c,(2.10),(2.2)$.

db) ) $\forall X(X\{\Gamma) \in M \rightarrow X \in P) \quad / d b A \rightarrow d b f . \quad$ dbA $) \quad \forall X ! X\{\Gamma) \in M$.

dbb) $\quad X\{\Gamma)_{\sigma M} / d b A,(3.2) . \quad$ dbc) $\quad X \in Q \quad / d b b, b$.

dbd) $X \Delta Q \quad / d b A, A . \quad$ dbe) $X=(\Delta \wedge \in) Q \quad /(2.3), d b d, d b c$.

dbf) $X \in P \quad / d b e, c$.

(5) Objects formed by satellites of an object

In this Section, we prove at first existence of objects formed by satellites of an object (defined in (5.1)). Next, we show existence of power objects of an object (defined in (5.2)), existence of sum objects of an object (defined in (5.3)), and existence of unit objects of an object (defined in (5.4)). We can prove that any object formed by satellites of an object, any power object of

15) In any proof of this type, we give a short sketch of a formal proof originally described in PLK together with its logical basis. 
an object, any sum object of an object, and any unit object of an object are all normal by (2.29) together with (3.5), (2.18), (2.12), (2.3), and (2.8). At the end of this Section, we prove further a proposition which can be regarded as a generalization of complete induction. (See (5.8).)

(5.1) $\exists P \cdot P\{\sigma) M$. (The relation $\{\sigma$ ) corresponds to the relation $\theta$ of $\mathbf{O Z}$. Compare $O Z$-(1.3.2) and $O Z$-(2.3.1). Accordingly, $P(\sigma) M$ is read " $P$ is an object formed by satellites of $M$ ". Proof. We can take an object, say $P$, satisfying $P\{=(\in \rightarrow \in) \wedge \sigma) M$ by (4.2). $P(\sigma) M$ can be proved by (2.3).)

(5.2) $\exists P \cdot P\{\subseteq) M$ as well as $\exists P \cdot P\{\subseteq) M . \quad(P\{\subseteq) M$ is read " $P$ is a power object of $M^{\prime \prime}$, as it means $\forall T(T \in P \equiv T \subseteq M)$. The first formula asserts existence of power object of any object. Compare $O Z$-(1.4.6). Proof. We can take two objects, say $Q$ and $R$, satisfying $Q\{=\subseteq \wedge \subseteq) M$ and $R\{=\subseteq \wedge \subseteq) M$ by (4.3). $Q\{\subseteq) M$ and $R\{\subseteq \subseteq) M$ can be proved by (2.2), (2.18), (2.22), and (2.23).)

(5.3) $\exists X \cdot X\{\hat{\epsilon} \in) Y . \quad(X\{\hat{\epsilon} \in) Y$ is read " $X$ is a sum object of $Y$ ". $\exists X \cdot X\{\in \in) Y$ seems unprovable in $\mathbf{O F}$. The theorem asserts existence of sum objects of any object. Compare $O Z$-(1.4.5). Proof. We can take an object, say $X$, satisfying $X\{=\in \in \wedge \sigma) Y$ by (4.2). $X\{\hat{\in} \in) Y$ can be proved by (1.2), (2.1), (2.2), (3.1), (3.2), and (3.5).)

(5.4) $\exists X \cdot X\{=) Y . \quad(X\{=) Y$ is read " $X$ is a unit object of $Y$ ". This interpretation may be natural, because $X\{=) Y$ means $\forall T(T \in X \equiv T=Y)$. However, it should be remarked here that $X\{=) Y$ is not a literal interpretation of $X\{Y\}$ of $\mathbf{O Z}$, as equality $=$ of $\mathbf{O F}$ is rather more closely related to proto-equality $\approx$ of $\mathbf{O Z}$ than identity $=$ of $\mathbf{O Z}$. (Compare (2.1) with $O Z$ (1.2.3) and $O Z$-(1.2.5).) The theorem asserts existence of unit objects of any object. Compare OZ-(1.4.4). Proof. We can take an object say $X$, satisfying $X\{==\wedge \sigma) Y$ by (4.2). $X\{=) Y$ can be proved by (2.1), (2.3), (2.17), (2.22), and (3.3).)

(5.5) $X\{=) Y \wedge U\{=) V \cdot \rightarrow \cdot X=U \equiv Y=V$. (The relation $\{=$ ) is unique in both directions. Proof. Assume $X\{=) Y$ and $U\{=) V$. Then, $X=U$ implies $Y=V$ by (2.2), (2.10), and (2.26); and $Y=V$ implies $X=U$ by (2.2), $(2.3),(2.7)$, and (2.11).) 
(5.6) $\exists X \cdot X\{\hat{E}) Y$. (Proof. By (1.2) and (4.5).)

(5.7) The relation $\{\sigma)_{\sigma}(\sigma\}$ implies inclusion $\subseteq$. Namely, $X\{\sigma)_{\sigma} Z \wedge Y\{\sigma) Z$. $\rightarrow X \subseteq Y$. (Compare $O Z-(2.3 .5)$. Proof. Assume $X\{\sigma) U_{\sigma} Z$ and $Y\{\sigma) Z$. Then, holds $X \subseteq Y$ by (2.15). Namely, for any member $T$ of $X$, holds $T \sigma U$, so also $T \sigma Z$ by (3.1), hence $T \in Y$.)

(5.8) $\forall X Y \cdot X(\Gamma \Gamma \vee \in \vee \subseteq \vee\{=) \cdot \rightarrow \Gamma) Y: \rightarrow U(\sigma \rightarrow \Gamma) V . \quad$ (Generalized complete induction: If membership $\in$ as well as the generalized inclusion $\subseteq$ as well as the unit-object relation $\{=$ ) implies a transitive relation $\Gamma$, the satellite relation $\sigma$ implies the relation $\Gamma$. Compare $O Z-(1.6)$, especially $O Z$ (1.6.1) and $O Z$-(1.6.4).)

Proof $/ A \rightarrow c$.

A) Assume $\forall X Y \cdot X(\Gamma \Gamma \vee \in \vee \leqq \vee\{=) \cdot \rightarrow \Gamma) Y$, i.e. assume that $\Gamma \Gamma$ implies $\Gamma$ and that $\in$ as well as $\subseteq$ as well as $\{=)$ implies $\Gamma$.

b)) $\quad \Gamma$ is left invariant $/ b A \rightarrow b d,(2.4) . \quad$ bA) $\forall X Y ! X=\Gamma Y$.

bb) $X \subseteq \Gamma Y \quad / b A,(2.17),(2.22)$ bc) $\quad X \Gamma \Gamma Y \quad / b d, A$.

bd) $X \Gamma Y / b c, A$.

c)) $\sigma$ implies $\Gamma \quad / c A \rightarrow c l$ cA) $\forall U V ! U_{\sigma} V$.

cb) $\exists P ! P\{=\Gamma \wedge \sigma) V \quad /(4.2) . \quad$ cc) $\quad P\{\Gamma \wedge \sigma) V \quad / c b, b,(2.2)$.

cd) ) $\forall S(S \in \in P \rightarrow S \subseteq \in P) \quad / c d A \rightarrow c d i . \quad \operatorname{cdA}) \quad \forall S ! S \in \in P$.

cdb) $\exists T ! S \in T \in P \quad / c d A . \quad$ cdc) $T \Gamma V \wedge T \sigma V / c d b, c c$.

cdd) $\quad S \Gamma T / c d b, A$ cde) $S \Gamma V / c d d, c d c, A$.

cdf) $\left.\quad S_{\sigma} T / c d b,(3.2) . \quad \mathbf{c d g}\right) \quad S_{\sigma} V / c d f, c d c,(3.1)$.

cdh) $S \in P \quad / c d e, c d g, c c . \quad$ cdi) $\quad S \subseteq \in P \quad / c d h,(2.13)$.

ce) $) \quad \forall S(S \subseteq \in P \rightarrow S \in \in P) \quad / c e A \rightarrow c e m . \quad$ ceA) $\quad \forall S ! S \subseteq \in P$.

ceb) $\exists T ! S \subseteq T \in P \quad / c e A . \quad$ cec) $T T V \wedge T \sigma V \quad / c e b, c c$.

ced) $\quad S \Gamma T / c e b, A$. cee) $\quad S_{\sigma} T / c e b,(3.3)$.

cef) $\exists R ! R\{=) S /(5.4)$. ceg) $R I S \quad / c e f, A$.

ceh) $\quad R \sigma S / c e f,(3.4) . \quad$ cei $) \quad R \Gamma V / c e g, c e d, c e c, A$.

cej) $\quad R \sigma V / c e h, c e e, c e c,(3.1) . \quad$ cek) $\quad R \in P \quad / c e i, c e j, c c$.

cel) $S \in R \quad / c e f,(2.26) . \quad$ cem) $\quad S \in \in P \quad / c e l, c e k$.

cf) $\sigma(P) / c d, c e,(1.4)$.

cg)) $\quad V \in \in P \quad$ lege, cgd. cga) $\exists W ! W\{=) V \quad /(5.4)$. 


$$
\begin{aligned}
& \text { cgb) } W \Gamma V \quad \operatorname{cga}, A . \quad \text { cge } \quad W \sigma V \quad \operatorname{cg} a,(3.4) \text {. } \\
& \text { cgd) } W \in P \quad / c g b, c g c, c c . \quad \text { cge) } \quad V \in W \quad / c g a,(2.26) \text {. } \\
& \text { ch) } U \in \in P \quad / c f, c g, c A,(1.5) \text {. } \\
& \text { ci) } \exists Z ! U \in Z \in P / c h . \quad \text { cj) } Z \Gamma V / c i, c c \text {. } \\
& \text { ck) } U I Z \quad / c i, A \text { cl) } U \Gamma V / c k, c j, A \text {. }
\end{aligned}
$$

(6) Null objects and pair objects

We can define null objects in OF in such a way that they have all the essental properties of the null set in ordinary set theories.

In $\mathbf{O F}$, we can further prove existence of suitably defined pair objects of any two objects. (See (6.9) and (6.11).) Of course, the definition of pair objects in OF turns out to be something different from the literal interpretation of the definition of pair objects in $\mathbf{O Z}$. It seems impossible in $\mathbf{O Z}$ to prove existence of pair objects of any two objects generally, neither in the sense of the definition of OZ-(3.2.9) nor in the sense of the literal interpretation of Definition (6.9).

(6.1) DF. $P \emptyset Q ₹ \forall S \cdot S \notin P$, and $\varnothing(P) \equiv \exists Q \cdot P \emptyset Q$. (Compare $O Z \cdot$ (1.4.1). $P \varnothing Q$ as well as $\varnothing(P)$ is read " $P$ is a null object". It should be noticed here that $P \varnothing Q$ is independent of $Q$ by definition. We introduce the relation $\varnothing$ because it seems sometimes convenient that we can denote the condition to be a null object in a form of a binary relation.

(6. 2) $\neg(X \in \emptyset Y)$. (Proof. By (6.1).)

(6.3) $X \varnothing Y \equiv X \varnothing Z$. (Proof. By (6.1).)

(6.4) The relation $\varnothing$ implies inclusion $\subseteq$, so holds $\varnothing(X) \rightarrow X \subseteq Y$. (Proof. By (2.15) and (6.1).)

(6.5) $\exists P \cdot \emptyset(P)$. (Existence of null objects. Compare $O Z-(1.4 .2)$. Notice that the following proof is quite different from the proof of $O Z$-(1.4.2).)

Proof $/ A \rightarrow g$. A) Denote the relation $\in \rightarrow \in$ by $\Gamma$.

b) $\exists P ! P\left\{(\Gamma)_{\sigma}\right) M$ /axiom. ( $M$ being an arbitrary object.)

c) $\forall S\left(S \in P \equiv S\{\Gamma)_{\sigma} M\right) \quad / b . \quad$ d) $\forall S\left(S \in P \equiv: \exists Z \cdot S\{\Gamma) Z_{\sigma} M\right) \quad / c$.

e) ) $\quad>\exists Z(S\{\Gamma) Z \sigma M) \quad / e A \rightarrow e e$ (reductio ad absurdum). 
eA) Assume $\exists Z ! S\{\Gamma) Z_{\sigma} M . \quad$ eb) $\quad \forall T(T \in S \equiv T \Gamma Z) \quad / e A$

ec) $\forall T(T \in S \equiv T(\in \rightarrow \in) Z) \quad / A, e b . \quad$ ed) $\quad \forall T \cdot T \in S \quad / e c$.

ee) contradiction $/ e d,(4.1)$.

f) $\forall S \cdot S \notin P \quad / d$, e. $\quad$ g) $\quad \varnothing(P) \quad / f,(6.1)$.

(6.6) $\neg X(\varnothing \wedge\{=)) Y$, i.e. $\quad \neg \exists X Y(\varnothing(X) \wedge X\{=) Y$ ). (Any null object can never be a unit object of an object. Proof. By (2.26) and (6.1).)

(6.7) The relation $\varnothing$ is invariant, and $X=Y \rightarrow \cdot \varnothing(X) \equiv \emptyset(Y)$. (Proof. By (2.1), (2.4), and (6.1).)

(6.8) $\varnothing(X) \wedge \varnothing(Y) \cdot \rightarrow X=Y$ and $X \varnothing U \wedge Y \varnothing V \cdot \rightarrow X=Y$. (Proof. By (2.1) and (6.1).)

(6.9) DF. $Z\{X, Y\} ₹ \forall T(T \in Z \equiv \cdot T=X \vee T=Y) . \quad(Z\{X, Y\}$ is read " $Z$ is a pair object of $X$ and $Y^{\prime \prime}$. Compare $O Z$-(3.2.9). Notice that $Z\{=) X$ can be also expressed as $Z\{X, X\}$.)

(6.10) $Z\{X, Y\} \rightarrow \cdot X \in Z \wedge Y \in Z$. (Proof. By (2.3) and (6.9).)

(6.11) $\exists Z \cdot Z\{X, Y\}$. (Existence of pair objects of any two objects. Compare $O Z$-(3.2.10). By virtue of this theorem, the theory of $\mathbf{O F}$ can be developed remarkably simpler than the theory of OZ.)
Proof $/ A, B \rightarrow c, d, e$;
(6.9).
A) $\forall M$ !

B) Define $\Gamma$ by $U \Gamma V ₹ \cdot(U \in X \wedge \varnothing(V)) \vee(U \in Y \wedge>\varnothing(V))$.

c) $\exists Z ! Z\left\{\{\Gamma)_{\sigma}\right) M \quad$ /axiom.

d) ) $\quad \forall T(T \in Z \rightarrow \cdot T=X \vee T=Y) \quad / d A \rightarrow d h . \quad$ dA) $\quad \forall T ! T \in Z$.

db) $\quad T\{\Gamma)_{\sigma Z} \quad / d A, c$.

de) $\exists S ! T\{\Gamma) S \sigma Z \quad / d b, \quad$ dd) $\quad \forall U(U \in T \equiv U \Gamma S) \quad / d c$.

de) $\forall U(U \in T \equiv \cdot(U \in X \wedge \varnothing(S)) \vee(U \in Y \wedge \neg \varnothing(S))) \quad / B, d d$.

df)) $\quad \varnothing(S) \rightarrow T=X \quad / d f A \rightarrow d f c . \quad$ dfA $\mathbf{A})$ Assume $\varnothing(S)$.

dfb) $\forall U(U \in T \equiv U \in X) \quad / d f A, d e . \quad$ dfc) $\quad T=X / d f b,(2.1)$.

dg) $) \quad \neg \varnothing(S) \rightarrow T=Y \quad / d g A \rightarrow d g c . \quad \operatorname{dgA}) \quad$ Assume $\neg \varnothing(S)$.

dgb) $\forall U(U \in T \equiv U \in Y) \quad / d g A, d e . \quad \operatorname{dgc}) \quad T=Y \quad / d g b,(2.1)$.

dh) $T=X \vee T=Y \quad / d f, d g$.

e)) $\quad \forall T(T=X \vee T=Y \cdot \rightarrow T \in Z) \quad / e a, e b$.

ea)) $\forall T(T=X \rightarrow T \in Z) \quad l e a A \rightarrow e a j . \quad$ eaA $) \quad \forall T ! \quad T=X$. 


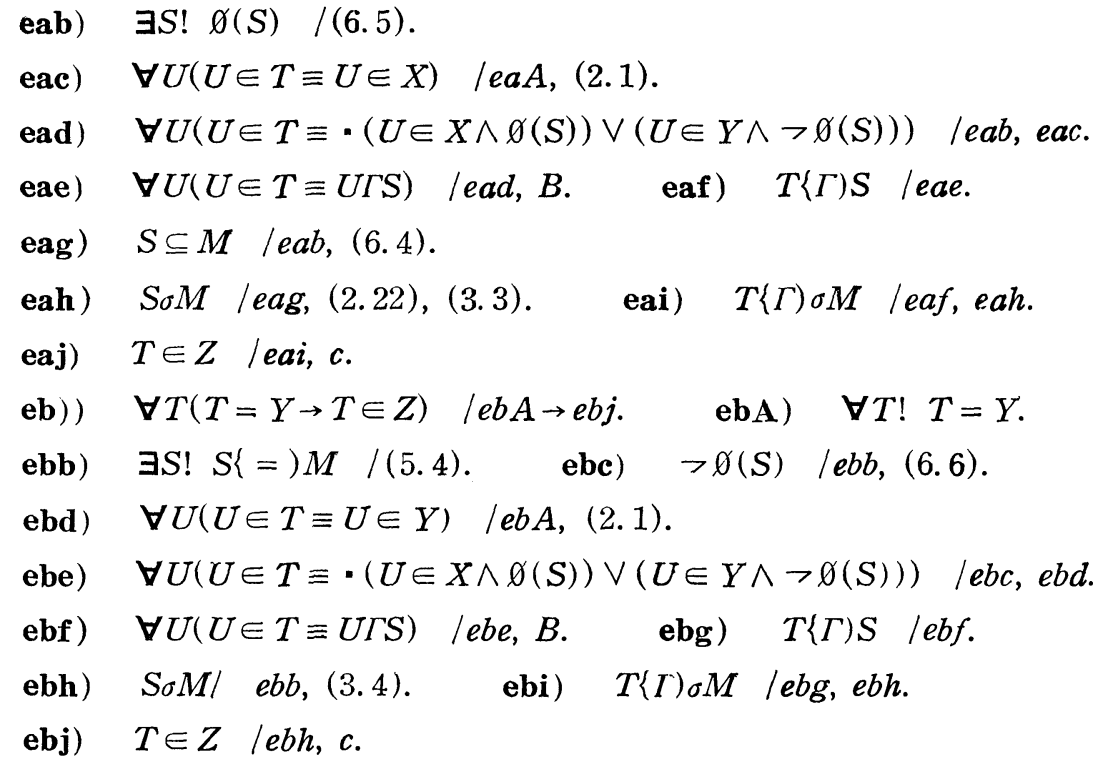

(6.12) $Z\{X, Y\} \rightarrow Z\{€) Z$. (Any pair object is normal. Proof. By (1.2), (2.1), (2.3), and (6.9).)

(6.13) $\exists Z \forall T(T \in Z \equiv \cdot T \hat{\in} X \vee T \hat{\in} Y$ ). (Any object $Z$ satisfying $\forall T(T \in Z$ $\equiv \cdot T \hat{\in} X \vee T \in \hat{E} Y$ ) can be regarded as a union object of $X$ and $Y$. Compare $O Z$-(3.2.11). Although it seems impossible to prove generally for any $X$ and $Y$ existence of an object $U$ in $\mathbf{O F}$ such that $\forall T(T \in U \equiv \cdot T \in X \vee T \in Y)$, we can develop the theory of $\mathrm{OF}$ in a remarkably simpler way than the theory of $\mathrm{OZ}$ because we can prove this theorem easily in OF. Proof. We can take a pair object, say $U$, of $X$ and $Y$ by (6.11). We can further take a sum

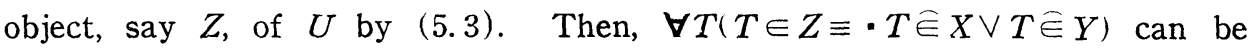
proved by (2.12), (6.9), and (6.10).)

(6. 14) $Z\{X, Y\} \wedge W\{U, V\} \cdot \rightarrow: Z=W \equiv \cdot(X=U \wedge Y=V) \vee(X=V \wedge Y=U)$. (Proof. By (2.3), (2.5), (6.9), and (6.10).)

(6.15) $X=Y \wedge X\{U, V\} \cdot \rightarrow Y\{U, V\}$. (Proof. By (2.1) and (6.9).)

\section{(7) Descendents}

Just as the notions of constituents and ancestors in $\mathbf{O Z}$, the notion of descendents is of particular importance in OF. By employing the notion of descendents, we can adequately define the notions, regularity and total 
normality, which are essentially important in OF. (See Sections (8) and (9).) In this Section, we define the notion of descendents (in (7.1)) and describe some fundamental properties of it. (Compare OZ-(1.5).)

(7.1) DF. $X \delta Y ₹ \forall P(\forall S(S \in \in P \rightarrow S \in P) \rightarrow(Y \in P \rightarrow X \in P))$. Any object $X$ satisfying $X \delta Y$ is called a descendent of $Y$.

Illustration. The notion of descendents of an object $Y$ in $\mathbf{O F}$ is a modification of the notion of descendents $x$ of a set $y$ in ordinary set-theories, which satisfy a condition of the form $x \in \cdots \in y$. The descendent relation $\delta$ is defined in such a way that $X \delta Y$ holds if and only if $X$ and $Y$ satisfy a condition of the form $X=\in \cdots \in Y$ for a finite number of $\in$ 's, including the case of no $\in$ 's. The adjustment is carried out in such a way that the relation $\delta$ becomes invariant. (See (7.6). Compare OZ-(1.5.1).)

(7.2) The descendent relation $\delta$ is reflexive and transitive. (Proof. By $(7.1)$.

(7.3) The generalized membership $E$ implies the descendent relation $\delta$. (Proof. By (1.2), (2.1), (2.2), (2.5), and (7.1).)

(7.4) Equality $=$ implies the descendent relation $\delta$. (Proof. By (2.2), (2.12) and (7.1).)

(7.5) The descendent relation $\delta$ implies the satellite relation $\sigma$. (Proof. Assume $X \delta Y Y$. To prove $X_{\sigma} Y$ by (1.5), take any object $Q$ satisfying $\sigma(Q)$ and $Y \in \in Q$. We can take an object $R$ satisfying $R\{=\subseteq \in \wedge \sigma) Q$ by (4.2). We can prove $R(\subseteq \in) Q$ by (2.2), (2.23), (3.1), (3.2), and (3.3). We can further prove $\forall S(S \in \in R \rightarrow S \in R$ ) by (1.2), (1.4), (2.1), (2.2), (2.6), (2.14), and (2.23); and $Y \hat{\in} R$ by (1.4) and (2.6). Hence $X \hat{\in} R$ by (7.1), which implies $X \in \in Q$ by (1.2), (1.4), (2.1), (2.2), and (2.23).)

(7.6) The descendent relation $\delta$ is invariant. (Proof. By (7.4), (7.2), and $(2.4)$.

(7.7) The descendent relation $\delta$ is equivalent to the relation $\delta \in V=$. (Compare OZ-(1.7.1). Proof. To prove that $\delta$ implies $\delta \in V=$, take any descendent $X$ of $Y$. We can take an object $P$ satisfying $P\{=(\delta \in \vee=) \wedge \sigma) Y$ by (4.2). We can prove $P\{\delta \in \vee=) Y$ by (2.2), (2.17), (2.22), (3.1), (3.2), (3.3), and (7.5), because $\delta \in \vee=$ is left invariant by (2.3), (2.9), and (7.6). 
We can further prove $\forall S(S \in \in P \rightarrow S \in P$ ) by (2.6), (7.2), (7.3), and (2.5); and $Y \hat{\in} P$ by (2.3) and (2.6). Hence holds $X \widehat{\in} P$ by (7.1) which implies $X(\delta \in \vee=) Y$ by (1.2) and (2.1). On the other hand, we can prove by (2.6), (7.3), (7.2), and (7.4) that $\delta \in \vee=$ implies $\delta$.)

\section{(8) Regularity}

We wish to call any object $M$ regular, if and only if $M$ has no infinite descending chain $M \ni X_{1} \ni X_{2} \ni \cdots$. However, our formal definition turns out to have a peculiar form by technical reason. (See (8.2), illustration.)

(8.1) DF. $M \rho N \vDash \forall X(X \delta M \wedge X \in N \cdot \rightarrow X \ni \in N)$, and $\rho(M) ₹ \neg M \rho \ni \delta M$. Any object $M$ satisfying $\rho(M)$ is called regular. (Compare $O Z-(2.2 .1)$.)

(8.2) Illustration. In ordinary set theories, $x \delta m$ can be interpreted as that $x$ and $m$ satisfy a condition of the form $x \in \cdots \in m$. (See (7.1), illustration.) Our Definition (8.1) of regularity may be justified, because, in the interpreted sense, $\forall m=\mu(m)$ is equivalent to the fundierung axiom assuming that the aussonderung axiom holds. This is shown informally as follows.

Namely, let $\Sigma$ be a set theory in which the aussonderung axiom holds. Then, we can take for any pair of sets $m$ and $n$, a set $p$ defined by $p=$ $\{x ; x \delta m \wedge x \in n\}$. If $x \delta m$ and $x \in n$ i.e. $x \in p$, then $x \ni \in n$ implies $x \ni \in p$ because $t \in \delta m$ implies $t \delta m$. Hence, $m \rho n$ is equivalent to $\forall x(x \in p \rightarrow x \ni \in p)$. On the other hand, if the fundierung axiom holds in $\Sigma$, then $m \rho n$ can be true only when $p$ is a null set. As $n \ni \delta m$ implies that $p$ is not a null set, $m \rho \ni \delta m$ can never hold. Thus, $\forall m \cdot \rho(m)$ can be proved. Next, let us assume conversely $\forall m \cdot \rho(m)$. Then, for any $p, p \ni \delta p$ implies $\neg p \rho p$. Evidently, $p \ni \delta p$ means that $p$ is non-void, and $\neg p \rho p$ implies that the set $p$ has a member disjoint with $p$. Hence, we have the fundierung axiom.

(8.3) $\emptyset(M) \rightarrow \rho(M)$. (Null object is regular. We can prove the theorem by reductio ad absurdum employing (6.1), (8.1), (7.7), and (6.7).)

(8.4) $K \delta M \wedge \rho(M) \cdot \rightarrow \rho(K)$. (Any descendent of a regular object is regular. Compare $O Z-(2.2 .5)$.)

Proof $/ A \rightarrow j . \quad$ A) Assume $K \delta M \wedge \neg \rho(K)$.

b) $\exists N S ! K \rho N \ni S \delta K / A,(8.1)$.

c) $\forall X(X \delta K \wedge X \in N \cdot \rightarrow X \ni \in N) \quad / b,(8.1)$. 

D) Define $\Gamma$ by $X \Gamma Y \geqslant X \delta K$.
e) $\exists L ! L\{=(\Gamma \wedge \in)) N /(4.4)$.
f)) $M_{\mu} L \quad / f A \rightarrow f k,(8.1)$.
fA) $\forall X ! X \delta M \wedge X \in L$.
fb) $\exists Y ! X=Y(\Gamma \wedge \in) N \quad / f A, e$.
fc) $Y \delta K \wedge Y \in N \quad / D, f b$.
fd) $\exists Z ! Y \ni Z \in N \quad / c, f c$.
fe) $\quad Z \in X \quad / f b, f d,(2.3),(2.5),(2.2)$.
ff) $\quad Z \delta Y \quad / f d,(2.6),(7.3)$
fg) $Z \delta K \quad / f f, f c,(7.2)$.
fh) $Z \Gamma N / f g, D . \quad$ fi) $Z=(\Gamma \wedge \in) N \quad /(2.3), f h, f d$.
fj) $Z \in L \quad / e, f i . \quad$ fk) $\quad X \ni \in L \quad / f e, f j$.
g) $\quad S \in L \quad / g b, e . \quad$ ga) $\quad S \Gamma N \quad / b, D$.
gb) $S=(\Gamma \wedge \in) N \quad /(2.3), g a, b$.
h) $S \delta M / b, A,(7.2)$ i) $M \rho \ni \delta M \quad / f, g, h$.
j) $>\rho(M) / i,(8.1)$.

(8.5) $K \in M \wedge \rho(M) \cdot \rightarrow \rho(K)$. (Any generalized member of a regular object is regular. Compare OZ-(2.2.5). Proof. By (8.4) and (7.3).)

(8.6) $M=N \rightarrow \cdot \rho(M) \equiv \rho(N)$. (Regularity is a property modulo equality. ${ }^{16)}$ Compare $O Z$-(1.2.6) and $O Z$-(2.2.5). It should be noticed here that $M=N$ in OF literally corresponds to $M \approx N$ in OZ. Proof. By (8.4), (7.4), and (2.3).)

(8.7) $\forall X(X \in M \rightarrow \rho(X)) \rightarrow \rho(M)$. (Any object formed by exclusively regular objects is itself regular. In $\mathbf{O Z}$, we have nothing corresponding to this particularly powerful theorem.)
Proof $/ A \rightarrow h$.
A) Assume $>\rho(M)$.
b) $\exists N S ! M \circ N \ni S \delta M / A,(8.1)$.
c) $\forall X(X \delta M \wedge X \in N \cdot \rightarrow X \ni \in N) \quad / b,(8.1)$.
d) $\exists T ! S \ni T \in N \quad / c, b$.
e) $\quad \delta \delta \in M \vee S=M \quad / b,(7.7)$.
f)) $\quad S \delta \in M \rightarrow \cdot \neg \forall X(X \in M \rightarrow \rho(X)) \quad / f A \rightarrow f f$.
fA) Assume $\exists U$ ! $S \delta U \in M$.
fb) $U \delta M / f A,(2.6),(7.3)$.
fc)) $U \rho N \quad / f c A \rightarrow f c c,(8.1) . \quad$ fcA) $\quad \forall X ! X \delta U \wedge X \in N$.
fcb) $X \delta M \quad / f c A, f b,(7.2) . \quad$ fcc) $\quad X \ni \in N \quad / c, f c b, f c A$.
fd) $U \rho \ni \delta U \quad / f c, b, f A$. fe) $\quad>\rho(U) \quad / f d,(8.1)$.
ff) $U \in M \wedge>\rho(U) \quad / f A, f e$.
g) ) $S=M \rightarrow \cdot \neg \forall ' X(X \delta M \rightarrow \rho(X)) \quad / g A \rightarrow g j . \quad$ gA) $\quad$ Assume $S=M$.

16) In the interpreted sense of $O Z \cdot(5.1 .1)$. 


$$
\begin{aligned}
& \text { gb) } T \delta S / d,(2.6),(7.3) \text { ge } \quad T \delta M \quad / g b, b,(7.2) \text {. } \\
& \text { gd) ) } T \rho N \quad \operatorname{gdA} \rightarrow g d c,(8.1) . \quad \operatorname{gdA}) \quad \forall X ! X \delta T \wedge X \in N \text {. } \\
& \text { gdb) } \quad X \delta M / g d A, g c,(7.2) \text {. gdc) } X \ni \in N \quad / g d A, g d b, c \text {. } \\
& \text { ge) } \quad T \ni \in N \quad / c, g c, d . \quad \text { gf) } \quad N \ni \delta T \quad / g e,(2.6),(7.3) \text {. } \\
& \text { gg) } T \rho \ni \delta T / g d, g f \text {. } \\
& \text { gh) }>\rho(T) \quad / g g,(8.1) . \quad \text { gi) } \quad T \in M \quad / d, g A,(2.5),(2.2) \text {. } \\
& \text { gj) } T \in M \wedge \neg \rho(T) \quad / g i, g h \text {. } \\
& \text { h) } \quad>\forall X(X \in M \rightarrow \rho(X)) \quad / e, f, g \text {. }
\end{aligned}
$$

(8.8) $K \subseteq M \wedge \rho(M) \cdot \rightarrow \rho(K)$. (Any generalized subobject of a regular object is regular. Compare $O Z-(2.2 .5)$. Proof. By (2.21), (8.5), and (8.7).)

(8.9) $K\{=) M \wedge \rho(M) \cdot \rightarrow \rho(K)$. (Any unit object of a regular object is regular. Compare OZ-(2.2.6). Proof. By (8.6) and (8.7).)

(8.10) $K \sigma M \wedge \rho(M) \cdot \rightarrow \rho(K)$. (Any satellite of a regular object is itself regular. Compare $O Z-(2.2 .7)$.)

Proof $/ A \rightarrow g$. A) Define $T$ by $X \Gamma Y \geqslant \cdot \rho(Y) \rightarrow \rho(X)$.
b) $\forall X Y \cdot X(I T \rightarrow \Gamma) Y \quad / A$.
c) $\forall X Y \cdot X(\in \rightarrow \Gamma) Y \quad /(2.6),(8.5), A$.
d) $\forall X Y \cdot X(\subseteq \rightarrow T) Y /(8.8), A$.
e) $\forall X Y \cdot X(\{=) \rightarrow \Gamma) Y /(8.9), A$.
f) $\forall X Y: X(\sigma \rightarrow \Gamma) Y \quad / b, c, d, e,(5.8)$.
g) $K \sigma M \wedge \rho(M) \cdot \rightarrow \rho(K) \quad / f, A$.

(8.11) $K(\sigma) M \wedge \rho(M) \cdot \rightarrow \rho(K)$. (Any object formed by satellites of a regular object is itself regular. Compare $O Z$-(2.3.2). In OF, we can prove this important theorem very easily by (8.10) and (8.7).)

(8.12) $R\{P, Q\} \wedge \rho(P) \wedge \rho(Q) \cdot \rightarrow \rho(R)$. (Any pair object of any two regular objects is itself regular. Proof. By (6.9), (8.6), and (8.7).)

\section{(9) Totally normal objects}

Together with regularity, total normality is a notion of particular importance in $\mathbf{O F}$. We call an object totally normal if and only if all the descendents of it are normal. (See (9.1).) In this Section, we show some elementary properties concerning total normality.

(9.1) DF. $\nu(P) ₹ \forall X(X \delta P \rightarrow X\{\hat{E}) X)$. Any object $P$ satisfying $\nu(P)$ is called totally normal. 
(9.2) $\nu(P) \rightarrow P\{\in) P$. (Totally normal objects are also normal. Proof. By (7.2) and (9.1).)

(9.3) $P=Q \rightarrow \cdot \nu(P) \equiv \nu(Q)$. (Total normality is a property modulo equality. Proof. By (9.1), (7.6), and (2.3).)

(9.4) $Q \delta P \wedge \nu(P) \cdot \rightarrow \nu(Q)$. Especially, $Q \in P \wedge \nu(P) \cdot \rightarrow \nu(Q)$. (Any descendent of a totally normal object is also totally normal. Especially, any generalized member of a totally normal object is also totally normal. Proof. By (7.2), (7.3), and (9.1).)

(9.5) $\forall X(X \in M \rightarrow \nu(X)) \wedge M\{\hat{E}) M \cdot \rightarrow \nu(M)$. (Any normal object formed exclusively by totally normal objects is totally normal.)

$$
\begin{aligned}
& \text { Proof } / A \rightarrow b . \quad \text { A) } \forall M ! \forall X(X \in M \rightarrow \nu(X)) \wedge M\langle\hat{\epsilon}) M \text {. } \\
& \text { b) ) } \quad \nu(M) \quad(b A \rightarrow b e,(9.1) . \quad \text { bA }) \quad \forall X ! X \delta M \text {. } \\
& \text { bb) } X \delta \in M \vee X=M \quad / b A \text { (7.7). } \\
& \text { bc)) } X \delta \in M \rightarrow X\{\hat{E}) X \quad / b c A \rightarrow b c e \text { bcA) Assume } X \delta \in M \text {. } \\
& \text { bcb) } \exists Y ! X \delta Y \in M \quad / b c A \text {. } \\
& \text { bcc) } \quad \nu(Y) \quad / b c b, A . \quad \text { bcd }) \quad \nu(X) \quad / b c b, b c c,(9.4) \text {. } \\
& \text { bce) } \quad X\{\hat{\epsilon}) X \quad / b c d,(9.2) \text {. } \\
& \text { bd) ) } X=M \rightarrow X\{\hat{\in}) X \quad(b d A \rightarrow b d c \text {. bdA) Assume } X=M \text {. } \\
& \text { bdb) } \quad X=\{\hat{E})=X \quad \text { (bdA, } A,(2.3) \text {. } \\
& \text { bde) } \quad X\{\hat{E}) X \quad /(2.2),(2.11),(2.12), b d b \text {. } \\
& \text { be) } \quad X\{€) X \quad / b b, b c, b d \text {. }
\end{aligned}
$$

(9.6) $Q\{\hat{E}) \subseteq P \wedge \nu(P) \cdot \rightarrow \nu(Q)$. (Any normal generalized subobject of a totally normal object is also totally normal. Naturally, $Q\{\hat{\in}) \subseteq P$ implies $Q\{\hat{E} Q$ by (2.12) and (2.29), and the same condition implies also $Q \subseteq P$ by (2.24) and (2.13). Proof of the theorem. For any totally normal $P$ and $Q$ satisfying $Q\{\hat{\epsilon}) Q$ and $Q \subseteq P$, we can prove $\forall X(X \in Q \rightarrow \nu(X))$ by $(2.14)$ and (9.4), so $Q$ is totally normal by (9.5).)

(9.7) $\varnothing(P) \rightarrow \nu(P)$. (Any null object is totally normal. Proof. By (1.2), (2.27), (2.28), (6.1), and (9.5).)

(9.8) $R\{P, Q\} \wedge \nu(P) \wedge \nu(Q) \cdot \rightarrow \nu(R), \quad$ especially $R\{=) P \wedge \nu(P) \cdot \rightarrow \nu(R)$. (Any pair object of any two totally normal objects is totally normal; especially, any unit object of a totally normal object is totally normal. We can prove 
the first formula by (6.9), (6.12), (9.3), and (9.5). As noticed in (6.9), $R\{P, P\}$ is equivalent to $R\{=) P$, so the first formula implies the second one.)

(9.9) $\exists P \cdot P\{\{\in) \subseteq) M$. (For any object $M$, there is an object formed by all the normal generalized subobjects of $M$. Proof. We can take an object $P$

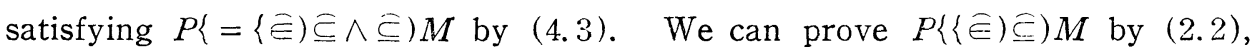
$(2.8),(2.10),(2.13)$, and $(2.24)$.)

(9.10) $P\{\{\in \in \subseteq) M \wedge \nu(M) \cdot \rightarrow \nu(P)$. (Any object formed by all the normal generalized subobjects of a totally normal object is also totally normal. Proof. By (2.8), (2.10), (2.29), (9.5), and (9.6).)

(9.11) $\nu(M) \rightarrow \cdot P\{\hat{\in} \in) M \equiv P\{\in \in) M$. (For any totally normal object $M$, $P\{\in \in) M$ and $P\{\in \in) M$ are mutually equivalent. Proof. By (9.4), (9.2), and (2.6).)

(9.12) $P\{\hat{\in} \in) M \wedge \nu(M) \cdot \rightarrow \nu(P)$. (Any sum object of any totally normal object is also totally normal. Proof. By (2.6), (2.8), (2.12), (2.29), (9.4), and (9.5).)

\section{(10) Natural numbers}

Although it is not indispensable to establish a full theory of natural numbers as a preparation for establishing a set theory, it seems adequate to introduce here a theory of natural numbers in our system OF by the interpretation that any null object is regarded as the number zero and any unit object of any natural number $X$ is regarded as the number following $X$. From this point of view, we write down here again a few propositions already stated. (See (10.8) and (10.9).) By proving that there is an object formed by all the natural numbers, we know that the axiom of infinity holds in OF in the sense that there is an object formed by infinitely many objects. To prove that we can construct a theory of sets in which the axiom of infinity in its original sense holds, we have to define the notion of sets in $O F$ and to show that any natural number as well as any object formed by all the natural numbers is a set. (See (11.13) and (11.14).)

Informally speaking, any object $X$ is called a natural number (notation " $\sharp(X)$ ") if and only if we can find $X$ in an infinite sequence $X_{0}, X_{1}, \ldots$, where $X_{0}$ is a null object and $X_{n+1}\{=) X_{n}$ holds for all $n$ (The notion of 
natural numbers is defined formally in (10.4).) For convenience' sake, we introduce a binary relation " $\neq$ " before defining natural numbers. The relation $=$ is naturally a relation closely connected with the notion of natural numbers. Namely, $X \pm Y$ means that we can find $X$ in an infinite sequence $Z_{0}, Z_{1}, \ldots$, where $Z_{0}$ is equal to $Y$ and $Z_{n+1}\{=) Z_{n}$ holds for all $n$. (See (10.1).) Accordingly, $X$ is a natural number if and only if $X=Y$ hoids for a null object $Y$. (See (10.4.) and (6.1).)

(10.1) DF. $X \sharp Y$ 羿 $\forall P(\forall S(S\{=) \in P \rightarrow S \in P) \rightarrow(Y \in P \rightarrow X \hat{\in}))$.

(10.2) The relation $\#$ is reflexive and transitive. It is also invariant. (Proof. By (2.3), (2.4), (2.12), and (10.1).)

(10.3) Equality = implies the relation \#. (Proof. By (10.1), (2.2) and (2.12).)

(10.4) DF. $\sharp(X) \equiv: \exists Y \cdot X \sharp \varnothing Y$. Any object satisfying $\sharp(X)$ is called a natural number.

(10.5) $\emptyset(X) \rightarrow \neq(X)$. (Zero is a natural number. In this Section, any null object is called "zero" for the purpose to make the interpreted images of our natural numbers vivid. Proof. By (6.1), (10.2), and (10.4).)

(10.6) The relation $\{=) \neq$ implies the relation $\sharp$. (Proof. Assume $X\{=) \# Y$. For any $P$ satisfying $\forall S(S\{=) \in P \rightarrow S \in P)$ and $Y \in P$, we can prove $X \in \mathcal{E}$ by (1.2), (2.1), (2.2), (2.3), (2.6), (2.11), and (10.1). So, $X=Y$ by (10.1).)

(10.7) $Y\{=) X \wedge \sharp(X) \cdot \rightarrow \sharp(Y)$. (Any unit object of a natural number is also a natural number. In this Section, we call any unit object of a natural number $X$ " a natural number following $X$ " for the same purpose as mentioned in (10.5). Proof. By (10.4) and (10.6).)

(10.8) $U\{=) X \wedge V\{=) Y \cdot \rightarrow \cdot X=Y \equiv U=V . \quad$ (See (5.5). Especially, in the field of natural numbers, any two numbers are mutually equal if and only if a natural number following one of them is equal to a number following the other.)

(10.9) $\varnothing(X) \rightarrow \cdot \neg X\{=) Y$. (Zero follows no natural number. See (6.6).)

(10.10) $\forall X(\varnothing(X) \rightarrow \mathfrak{I}(X))$ and $\forall Y Z((Z=Y \vee Z\{=) Y) \wedge \mathfrak{A}(Y) \cdot \rightarrow \mathfrak{A}(Z))$ imply $\forall W(\sharp(W) \rightarrow \mathfrak{H}(W)$ ). (This theorem corresponds to the complete induction. Evidently, the second assumption of the theorem can be replaced 
by $\forall Y Z(Z\{=) Y \wedge \mathfrak{P}(Y) \cdot \rightarrow \mathfrak{H}(Z))$ for any condition $\mathfrak{\Re}(T)$ on $T$ modulo equality. Compare $O Z$-(1.8.11) and $O Z$-(6.9.10).)

Proof $/ A \rightarrow b$.

A) Assume $\forall X(\varnothing(X) \rightarrow \mathfrak{U}(X))$ and $\forall Y Z((Z=Y \vee Z\{=) Y) \wedge \mathfrak{A}(Y) \cdot \rightarrow \mathfrak{U}(Z))$.

b) ) $\forall W(\sharp(W) \rightarrow \mathfrak{A}(W)) \quad / b A \rightarrow b d . \quad$ bA) $\quad \forall W ! \sharp(W)$.

bb) $\exists U V ! W \sharp U \varnothing V / b A,(10.4)$.

bc) $\forall P(\forall S(S\{=) \in P \rightarrow S \in P) \rightarrow(U \in P \rightarrow W \in P)) \quad / b b,(10.1)$.

bd)) $\quad \mathfrak{H}(W) \quad / b d A \rightarrow b d k . \quad$ bdA $) \quad$ Define $\Gamma$ by $F \Gamma G \stackrel{2}{2}(F)$.

bdb) $\exists P ! P\{=\Gamma \wedge \sigma) U /(4.2)$.

bde) $\quad \forall S(S\{=) \in P \rightarrow S \in P) \quad(b d c \mathrm{~A} \rightarrow b d c n . \quad$ bdeA $) \quad \forall S ! S\{=) \in P$.

bdcb) $\exists T ! S\{=) T \in P \quad / b d c A . \quad$ bdcc) $T=\Gamma U \wedge T_{\sigma} U \quad / b d c b, b d b$.

bded) $\exists R ! T=R \Gamma U$ lbdcc. bdce) $\exists Q ! Q\{=) R /(5.4)$.

bdef) $S=Q \quad / b d c b, b d c e, b d c d,(10.8) . \quad$ bdeg) $\mathfrak{A}(R) \quad / b d c d, b d A$.

bdch) $\quad \mathfrak{H}(Q) \quad / b d c g, b d c e, A . \quad$ bdci $) \quad Q \Gamma U / b d c h, b d A$.

bdcj) $\quad S=\Gamma U \quad / b d c f, b d c i$.

bdck) $S=\{=)=\sigma U \quad / b d c f, b d c e, b d c d, b d c c,(2.3)$.

bdcl) $\quad S \sigma U$ /bdck, (2.3), (2.11), (2.2), (3.4), (3.1).

bdem $) \quad S(=\Gamma \wedge \sigma) U \quad / b d c j, b d c l . \quad$ bden $) \quad S \in P \quad / b d c m, b d b$.

bdd)) $\quad U \in P \quad / b d d g,(2.6)$.

bdda) $\quad \varnothing(U) \quad / b b,(6.1) . \quad$ bddb $) \quad \mathfrak{H}(U) \quad / b d d a, A$.

bddc) $\quad U T U / b d d b, b d A$. bddd) $U=\Gamma U \quad / b d d c,(2.3)$.

bdde) $\quad U_{\sigma} U /(3.1) . \quad$ bddf $) \quad U(=\Gamma \wedge \sigma) U / b d d d, b b d e$.

bddg) $\quad U \in P \quad / b d d f, b d b$.

bde) $\quad W \in P \quad / b c, b d c, b d d . \quad$ bdf) $\quad \exists M ! W=M \in P \quad / b d e,(1.2),(2.1)$.

bdg) $\quad M=\Gamma U \quad / b d f, b d b . \quad$ bdh) $\quad W=\Gamma U \quad / b d f, b d g,(2.3)$.

bdi) $\exists K ! W=K T U / b d h . \quad$ bdj) $\mathfrak{A}(K) \quad / b d i, b d A$.

bdk) $\quad \mathfrak{H}(W) \quad / b d i, b d j, A$.

(10.11) $\sharp(W) \rightarrow \rho(W)$. (Every natural number is regular.)

Proof $/ A \rightarrow d . \quad$ A) Assume $\sharp(W)$. b) $\forall X(\varnothing(X) \rightarrow \rho(X)) \quad /(8.3)$.

c) $\quad \forall Y Z((Z=Y \vee Z\{=) Y) \wedge \rho(Y) \cdot \rightarrow \rho(Z) \quad /(8.6),(8.9)$.

d) $\rho(W) / A, b, c,(10.10)$.

(10.12) $\sharp(W) \rightarrow \nu(W)$. (Every natural number is totally normal.) 
Proof $/ A \rightarrow d . \quad$ A) Assume $\sharp(W)$. b) $\forall X(\varnothing(X) \rightarrow \nu(X)) \quad /(9.7)$.

c) $\forall Y Z((Z=Y \vee Z\{=) Y) \wedge \nu(Y) \cdot \rightarrow \nu(Z)) \quad /(9.3),(9.8)$.

d) $\nu(W) / A, b, c,(10.10)$.

(10.13) $\#$-relation implies $\sigma$-relation. (Proof. For any $X$ and $Y$ satisfying $X \pm Y$, take an object $P$ satisfying $P\{\sigma) Y$ by (5.1). Then, we can prove $\forall S(S\{=) \in P \rightarrow S \in P$ ) by (3.1) and (3.4). Since $Y \in P$ by (3.1) and (2.6), we can prove $X \sigma Y$ by (1.2), (2.1), (2.2), (3.5), and (10.1).)

(10.14) $\exists P \cdot P(\sharp) M . \quad$ (Proof. By (2.2), (4.2), (10.2), and (10.13).)

(10.15) $\exists P \cdot P\{\emptyset) M$, i.e. $\exists P \forall S(S \in P \equiv \pm(S))$. (There is an object formed by all the natural numbers. Compare $O Z$-(1.8.5) and $O Z$-(6.9.6). Proof. By (6.5) and (10.14), we can take objects $U$ and $P$ satisfying $\varnothing(U)$ and $P(\Psi) U$. For this $P$, we can prove $P\{ \pm \varnothing) M$ and $\forall S(S \in P \equiv \#(S))$ by (2.2), (6.1), (6.3), (6.8), (10.2), and (10.4).)

(10.16) $P\{\because \varnothing) M \rightarrow \rho(P)$. (Any object formed by all the natural numbers is regular.)

Proof $/ A \rightarrow g . \quad$ A) Assume $P\{\because \varnothing) M$ b) $\exists U ! \varnothing(U) /(6.5)$.

c) $\exists Q ! Q\{\sigma) U /(5.1)$.

d) ) $P \subseteq Q \quad / d A \rightarrow d f,(2.15) . \quad$ dA) $\quad \forall T ! T \in P$.

db) $T \leftleftarrows \varnothing M / A, d A . \quad$ de) $T \leftleftarrows \varnothing U / d b,(6.3)$.

dd) $T \sigma \sigma U / d c,(10.13),(6.4),(2.22),(3.3)$.

de) $T_{\sigma} U / d d,(3.1) . \quad$ df) $T \in Q \quad / c$, de.

e) $\rho(U) \quad / b,(8.3) . \quad$ f) $\rho(Q) \quad / c, e,(8.11)$.

g) $\rho(P) \quad / d, f,(2.22),(8.8)$.

(10.17) $P(\sharp \varnothing) M \rightarrow \nu(P)$. (Any object formed by all the natural numbers is totally normal.)
Proof $/ A \rightarrow d$.
A) $\forall P M ! P\{\amalg \varnothing) M$.
b)) $\forall X(X \in P \rightarrow \nu(X)) \quad(b A \rightarrow b d . \quad$ bA $) \quad \forall X ! X \in P$.
bb) $X \sharp 0 M \quad / b A, A$.
bc) $\sharp(X) \quad / b b,(10.4) . \quad$ bd) $\quad \nu(X) \quad / b c,(10.12)$.
c) $P\{\in \mid P \quad / A,(10.2),(2.8),(2.29) . \quad$ d) $\quad \nu(P) \quad / b, c,(9.5)$.

(10.18) $V \delta W \wedge \sharp(W) \cdot \rightarrow \sharp(V)$, i.e. the relation $\delta \sharp \varnothing$ implies the relation $\sharp \varnothing$. 
(Any descendent of a natural number is also a natural number. Since the second statement can be easily deduced from the first formula by (6.3) and (10.4), we prove here the first formula formally.)

Proof $/ a-f$.

a)) $\forall X(\emptyset(X) \rightarrow \forall T(T \delta X \rightarrow \sharp(T))) \quad / a A \rightarrow a b . \quad$ aA $) \quad \forall X ! \varnothing(X)$.

ab) ) $\forall T(T \delta X \rightarrow \sharp(T)) \quad / a b A \rightarrow a b f . \quad$ abA) $\forall T$ ! $T \delta X$.

abb) $\quad T \delta \in X \vee T=X \quad / a b A,(7.7) . \quad$ abc) $\quad>T \delta \in X \quad / a A,(6.1)$.

abd) $\quad T=X \quad / a b b, a b c . \quad$ abe) $\quad \varnothing(T) \quad / a A, a b d,(6.7)$.

abf) $\quad \Psi(T) \quad$ labe, (10.5).

b)) $\quad \forall Y Z(Z=Y \wedge \forall T(T \delta Y \rightarrow \sharp(T)) \cdot \rightarrow \forall T(T \delta Z \rightarrow \sharp(T))) \quad / b A \rightarrow b b$.

bA) $\forall Y Z ! Z=Y \wedge \forall T(T \delta Y \rightarrow \sharp(T))$.

bb) $) \quad \forall T(T \delta Z \rightarrow \sharp(T)) \quad / b b A \rightarrow b b d . \quad$ bbA $\quad \forall T ! T \delta Z$.

bbb) $\quad T \delta=Y \quad / b b A, b A . \quad$ bbc) $\quad T \delta Y \quad / b b b,(2.2),(7.6)$.

bbd) $\sharp(T) \quad / b b c, b A$.

c)) $\quad \forall Y Z(Z\{=) Y \wedge \forall T(T \delta Y \rightarrow \sharp(T)) \cdot \rightarrow \forall T(T \delta Z \rightarrow \sharp(T))) \quad / c A \rightarrow c b$.

cA) $\forall Y Z ! Z\{=) Y \wedge \forall T(T \delta Y \rightarrow \Psi(T))$.

cb) ) $\quad \forall T(T \delta Z \rightarrow \sharp(T)) \quad / c b A \rightarrow c b e . \quad$ cbA $) \quad \forall T$ ! $T \delta Z$.

cbb) $\quad T \delta \in Z \vee T=Z \quad / c b A,(7.7)$.

cbc)) $\quad T \delta \in Z \rightarrow \sharp(T) \quad / c b c A \rightarrow c b c e . \quad \operatorname{cbcA}) \quad$ Assume $\exists S ! T \delta S \in Z$.

cbcb) $\quad S=Y \quad / c b c A, c A . \quad$ cbcc) $\quad T \delta=Y \quad / c b c A, c b c b$.

cbed) $\quad T \delta Y \quad / c b c c,(2.2),(7.6) . \quad$ cbce $) \quad \sharp(T) \quad / c b c d, c A$.

cbd) ) $\quad T=Z \rightarrow \sharp(T) \quad / c b d A \rightarrow c b d e . \quad \operatorname{cbd} \mathbf{A}) \quad$ Assume $T=Z$.

cbdb) $\quad Y \delta Y /(7.2)$.

cbde) $\quad \sharp(Y) \quad / c A, c b d b . \quad$ cbdd) $\quad \sharp(Z) \quad / c b d c, c A,(10.7)$.

cbde) $\quad(T) \quad / c b d d, c b d A,(10.2),(10.4),(2.2)$.

cbe) $\sharp(T) \quad / c b b, c b c, c b d$.

d) $\forall Y Z((Z=Y \vee Z\{=) Y) \wedge \forall T(T \delta Y \rightarrow \sharp(T)) \cdot \rightarrow \forall T(T \delta Z \rightarrow \sharp(T))) \quad / b, c$.

e) $\sharp(W) \rightarrow \forall T(T \delta W \rightarrow \sharp(T)) \quad / a, b,(10.10)$.

f) $\quad V \delta W \wedge \sharp(W) \cdot \rightarrow(V) \quad / e$.

(10.19) Remark. The relation $\delta$ can be regarded as the relation " $\leq$ " in the field of natural numbers. However, we do not discuss the matter here in detail. (Compare OZ-(6.9.14).) 


\section{(11) Sets and set variables}

To construct a set theory in $\mathbf{O F}$, our field of objects has only to be restricted to a special field, the field of sets. Just as in $\mathbf{O Z}$ or in other theories of classes, it seems adequate to introduce set variables also in OF. In this Section, we try to define sets in such a way that a theory of sets can be imbedded in OF.

(11.1) DF. $\$(X) ₹ \cdot \rho(X) \wedge \nu(X)$. Any object $X$ satisfying $\$(X)$ i.e. any object which is regular and totally normal is called a set.

(11.2) Set variables. We use small Latin letters for denoting sets. Expressions of the forms $\forall s \cdot \widetilde{F}(s)$ and $\exists s \cdot \widetilde{F}(s)$ naturally denote $\forall S(S(S) \rightarrow \widetilde{F}(S))$ and $\exists S(S(S) \wedge \mathfrak{F}(S))$ respectively. Simple nominations of the form Qs! $\mathfrak{F}(s)$ in PLK naturally denote QS! $S(S) \wedge \mathfrak{F}(S)$. General nominations of the form $\forall s \mathbf{Q}_{1} t_{1} \cdots \mathbf{Q}_{k} t_{k} ! \widetilde{F}\left(s, t_{1}, \ldots, t_{k}\right)$ and general nominations of the form $\exists s \mathbf{Q}_{1} t_{1} \cdots \mathbf{Q}_{k} t_{k} ! \Im\left(s, t_{1}, \ldots, t_{k}\right)$ in PLK naturally denote the series of expressions defined recursively by $\forall S ! S(S), \mathbf{Q}_{1} t_{1} \ldots \mathbf{Q}_{k} t_{k} ! \mathfrak{F}\left(S, t_{1}, \ldots, t_{k}\right)$ and $\exists S ! \quad \$(S) \wedge \mathbf{Q}_{1} t_{1} \cdots \mathbf{Q}_{k} t_{k} \cdot \widetilde{F}\left(S, t_{1}, \ldots, t_{k}\right), \quad \mathbf{Q}_{1} t_{1} \cdots \mathbf{Q}_{k} t_{k} ! \tilde{F}\left(S, t_{1}, \ldots, t_{k}\right)$, respectively. Here in these expressions, the variables $s$ and $S$ are assumed to occur only in the indicated places; each one of $\mathbf{Q}, \mathbf{Q}_{1}, \ldots, \mathbf{Q}_{k}$ denotes either of the quantifier symbols $\forall$ or $\exists ;$ and $t_{1}, \ldots, t_{k}$ may be set variables or object variables $(k \geq 1), \quad \mathbf{Q}_{1} \mathrm{t}_{1} \cdots \mathbf{Q}_{j} \mathrm{t}_{j} \mathbf{Q} s \cdot \mathfrak{F}\left(\mathrm{t}_{1}, \ldots, \mathrm{t}_{j}, s\right)$ and $\mathbf{Q}_{1} \mathrm{t}_{1} \ldots$ $\left.\mathbf{Q}_{j}\right\rfloor_{j} \mathbf{Q}_{s} \mathbf{Q}_{j+1} t_{j+1} \cdots \mathbf{Q}_{j+k} t_{j+k} ! \widetilde{F}\left(t_{1}, \ldots, t_{j}, s, t_{j+1}, \ldots, t_{j+k}\right)(j, k \geq 0)$ are called the expressions obtained by restricting the quantifiers $\mathbf{Q S}$ of $\mathbf{Q}_{1} t_{1} \cdots \mathbf{Q}_{j} t_{j} \mathbf{Q S}$. $\widetilde{F}\left(t_{1}, \ldots, t_{j}, S\right)$ and $\mathbf{Q}_{1} t_{1} \cdots \mathbf{Q}_{j} t_{j} \mathbf{Q S Q}_{j+1} \dagger_{j+1} \cdots \mathbf{Q}_{j+k} \mathrm{t}_{j+k} ! \widetilde{F}\left(\mathrm{t}_{1}, \ldots, \mathrm{t}_{j}, \mathrm{~S}, \mathrm{t}_{j+1}\right.$, $\left.\ldots, t_{j+k}\right)$ to the set range, respectively. (Compare $O Z$-(5.2.1) and see $P D$-(1). Notice that $\forall s ! \mathfrak{F}(s)$ does not denote $\forall S ! S(S) \rightarrow \widetilde{F}(S)$, but does denote $\forall S$ ! $\$(S) \wedge \widetilde{\jmath}(S)$.)

Obviously, $\forall S \cdot \mathfrak{F}(S)$ implies $\forall s \cdot \mathfrak{F}(s)$ and $\exists s \cdot \mathfrak{f}(s)$ implies $\exists S \cdot \mathfrak{F}(S)$. In the following, however, we do not expressly refer to this Paragraph when we make use of this property.

(11.3) The set-theoretical images of formulas and the set-theoretical images of special predicates and relations. Just as in $\mathbf{O Z}$, we introduce notations of the form $|\mathfrak{x}|$, which denotes the formula obtained by restricting all the quantifiers (including nominating quantifiers) of the formula $\mathfrak{i}$ to the set range, and we call $|\mathfrak{A}|$ the set-theoretical image of the formula $\mathfrak{A}$. (Compare $O Z$ - 
(5.3.2) and $O Z$-(5.3.5).) If $\Gamma$ is a special symbol such as " $\subseteq$ " or " $\rho$ ", we denote sometimes $|X \Gamma Y|$ by $X \hat{\Gamma} Y$ and $|\Gamma(X)|$ by $\hat{\Gamma}(X)$. For example $|X \subseteq Y|$ as well as $X \widehat{\subseteq} Y$ denotes the same formula $\forall s(s \in X \rightarrow s \in Y)$. We can regard $\hat{\Gamma}$ as a special relation or a special predicate when $\Gamma$ denotes a special relation or predicate. Relations or conditions of this type are expressed simply by attaching the word "set-theoretical". For example, " $X \subseteq \widehat{\subseteq} Y$ " is read " $X$ is a set-theoretical subobject of $Y$ " or " $X$ is set-theoretically included in $Y$ " and " $\hat{\rho}(X)$ " is read " $X$ is set-theoretically regular".

We have some obvious rules for constructing set-theoretical images of formulas such as " $|\mathfrak{A} \wedge \mathfrak{B}|$ is $|\mathfrak{U}| \wedge|\mathfrak{B}|$ ", " $|\forall X \cdot \mathfrak{U}(X)|$ is $\forall x|\mathfrak{H}(x)|$, assuming that no more $X$ 's and $x$ 's occur in $\mathfrak{H}(T)$ ", etc. However, we do not discuss the matter here in detail. (Compare OZ-(5.3.4).)

(11.4) $\$(x), \rho(x)$, and $\nu(x)$. (Any set is a set. Any set is regular and totally-normal. Proof. By (11.1) and (11.2).)

(11.5) $\varnothing(P) \rightarrow \$(P)$. (Any null object is a set. Accordingly, null objects can be called also null sets. Proof. By (8.3), (9.7), and (11.1).)

(11.6) $X=y \rightarrow \$(X)$. (Any object which is equal to a set is also a set. Namely, the condition $\$(T)$ is a condition on $T$ modulo equalty. Compare $O Z$-(5.1.5).)

Proof $/ A \rightarrow d . \quad$ A) Assume $X=y . \quad$ b) $\quad \rho(y) \wedge \nu(y) /(11.4)$.

c) $\rho(X) \wedge \nu(X) \quad / A, b,(8.6),(9.3) . \quad$ d) $\$(X) \quad / c,(11.1)$.

(11.7) $X \hat{\in} y \rightarrow \$(X)$. (Any generalized member of a set is also a set. Compare $O Z$-(5.1.14).)
Proof $/ A \rightarrow d$.
A) Assume $X \hat{\in} y$.
b) $\rho(y) \wedge \nu(y) \quad /(11.4)$.
c) $\rho(X) \wedge \nu(X) \quad / A, b,(8.5),(9.4)$.
d) $\$(X) / c,(11.1)$.

(11.8) $X\{=) y \rightarrow \$(X)$. (Any unit object of a set is also a set. See (11.11).)

Proof $/ A \rightarrow d . \quad$ A) Assume $X\{=) y . \quad$ b) $\quad \rho(y) \wedge \nu(y) \quad /(11.4)$.

c) $\rho(X) \wedge \nu(X) \quad / A, b,(8.9),(9.8)$.

d) $\$(X) / c,(11.1)$.

(11.9) $P\{\{\hat{E}) \subseteq$ ) $m \rightarrow \$(P)$. (Any object formed by normal generalized subobjects of a set is also a set. Compare $O Z-(3.2 .5)$.) 
Proof $/ A \rightarrow h . \quad$ A) Assume $P\{\{(\hat{E}) m$.

b) $\rho(m) \wedge \nu(m) /(11.4)$.

c) $\exists Q ! Q\{\sigma) m /(5.1) . \quad$ d) $\rho(Q) \quad / c, b,(8.11)$.

e) $\quad P \subseteq Q \quad l e A \rightarrow e e,(2.15) . \quad$ eA) $\forall T ! T \in P$.

eb) $T\{\hat{E}) \subseteq m / A, e A$.

ec) Toom $l e b,(2.24),(3.3) . \quad$ cd) Tom lec, (3.1).

ee) $T \in Q \quad / c, e d$.

f) $\quad \rho(P) \quad / \boldsymbol{e}, d,(2.22),(8.8) . \quad$ g) $\quad \nu(P) \quad / A, b,(9.10)$.

h) $\$(P) \quad / f, g,(11.1)$.

(11.10) $P\{\in \in) m \rightarrow \$(P)$ and $Q\{\hat{\in} \in) m \rightarrow \$(Q)$. (The second formula asserts that any sum object of a set is also a set. Since $Q\{\hat{\epsilon} \in) m$ is equivalent to $Q\{\in \in) m$ by (9.11) and (11.4), we have only to prove the first formula. Compare OZ-(3.2.5).)

Proof $/ A \rightarrow h . \quad$ A) Assume $P\{\in \in) m$. b) $\rho(m) \wedge \nu(m) /(11.4)$.

c) $\exists R ! R\{\sigma) m /(5.1) . \quad$ d) $\rho(R) \quad / c, b,(8.11)$.

e) $\quad P \subseteq R \quad l e A \rightarrow e e,(2.15) . \quad$ eA $) \quad \forall T ! T \in P$.

eb) $T \in \in m / A, e A$. ec) $T$ oom /eb, (3.2).

ed) Tom lec, (3.1). ee) $T \in R \quad / c, e d$.

f) $\quad \rho(P) \quad / e, d,(2.22),(8.8) . \quad$ g) $\quad \nu(P) \quad / A, b,(9.11),(9.12)$.

h) $\$(P) \quad / f, g,(11.1)$.

(11.11) $R\{p, q\} \rightarrow \$(R)$. (Any pair object of any two sets is also a set. According to $(6.9),(11.8)$ is a special case of this theorem. Compare $O Z$ (3.2.11).)

Proof $/ A \rightarrow d . \quad$ A) Assume $R\{p, q\}$.

b) $\quad \rho(p) \wedge \nu(p)$ and $\rho(q) \wedge \nu(q) \quad /(11.4)$.

c) $\rho(R) \wedge \nu(R) \quad / A, b,(8.12),(9.8) . \quad$ d) $\quad \$(R) \quad / c,(11.1)$.

(11.12) $X \delta y \rightarrow \$(X)$. (Any descendent of a set is also a set.)
Proof $/ A \rightarrow d$.
A) Assume $X \delta y$.
b) $\rho(y) \wedge \nu(y) \quad /(11.4)$.

c) $\rho(X) \wedge \nu(X) \quad / A, b,(8.4),(9.4)$.

d) $\$(X) / c,(11.1)$.

(11.13) $\sharp(W) \rightarrow \$(W)$. (Any natural number is a set. Proof. By (10.11), (10.12), and (11.1).) 
(11.14) $P\{\# \varnothing) M \rightarrow \$(P)$. (Any object formed by all the natural numbers is a set. Proof. By (10.16), (10.17), and (11.1).)

(11.15) $\forall X(X \in M \rightarrow \$(X)) \wedge M\{€) M \cdot \rightarrow \$(M)$. (Any normal object formed exclusively by sets is itself a set.)
Proof $/ A \rightarrow f$.
A ) Assume $\forall X(X \in M \rightarrow \$(X)) \wedge M\{\hat{E}) M$.
b) $\forall X(X \in M \rightarrow \rho(X)) \quad / A,(11.1)$.
c) $\rho(M) \quad / b,(8.7)$.
d) $\forall X(X \in M \rightarrow \nu(X)) \quad / A,(11.1)$.
e) $\nu(M) / d, A,(9.5)$.
f) $\$(M) / c, e,(11.1)$.

\section{(12) Extensionality}

To construct a set theory in OF, it is desirable that sets of the same extent can be regarded as equal to each other. In this Section, we describe some theorems concerning the extensionality of sets in preparation for constructing a theory of sets. (See especially (12.4) and (12.9).)

(12.1) $X=Y \rightarrow \forall p(X \in p \equiv Y \in p)$. (Equal objects are either simultaneously members of a set or simultaneously non-members of a set.)

Proof $\mid A \rightarrow d . \quad$ A) Assume $X=Y$.

b)) $\forall p(X \in p \rightarrow Y \in p) \quad(b A \rightarrow b e . \quad$ bA $) \quad \forall p ! X \in p$.

bb) $\quad \nu(p) /(11.4)$. be $p\{\hat{\epsilon}) p \quad / b b,(9.2)$.

bd) $\quad Y € p \quad / A, b A,(2.3),(1.2),(2.1)$ be) $\quad Y \in p \quad / b c, b d$.

c) $\forall p(Y \in p \rightarrow X \in p)$ / similarly to $b$.

d) $\forall p(X \in p \equiv Y \in p) \quad / b, c$.

(12.2) $\forall P(X \in P \rightarrow Y \in P) \rightarrow X=Y$. (If $Y$ is a member of every object containing $X$ as its member, $X$ and $Y$ are mutually equal.)

Proof $/ A \rightarrow e . \quad$ A) Assume $\forall P(X \in P \rightarrow Y \in P)$.
b) $\exists Q ! Q\{=) X \quad /(5.4)$.
c) $X \in Q \quad / b,(2.26)$.
d) $Y \in Q \quad / A, c$.
e) $X=Y \quad / b, d,(2.3)$.

(12.3) $\forall p(x \in p \rightarrow Y \in p) \rightarrow x=Y$. (If $Y$ is a member of every set containing a set $x$ as its member, $x$ and $Y$ are mutually equal.)

\section{Proof $/ A \rightarrow f . \quad$ A) Assume $\forall p(x \in p \rightarrow Y \in p)$.}

b) $\exists Q ! Q\{=) x /(5.4)$. 
c) $x \in Q \quad / b,(2.26) . \quad$ d) $\quad \$(Q) \quad / b,(11.8)$.

e) $Y \in Q \quad / A, c, d,(11.2)$.

f) $x=Y \quad i b, e,(2.3)$.

(12.4) $x=y \equiv \forall p(x \in p \equiv y \in p)$. (Two sets are equal to each other if and only if they are either simultaneously members or simultaneously no members of every set. Proof. By (12.1) and (12.3).)

(12.5) $X \in y \equiv X \hat{\in} y . \quad$ (Broadly speaking, membership $\in$ is equivalent to the generalized membership $\hat{\in}$ for sets. Proof. By (9.2) and (11.4).)

(12.6) $X\{\hat{E}) y \equiv X=y$. (Broadly speaking, the relation $\{\hat{E})$ is equivalent to equality $=$ for sets. Proof. By (2.1) and (12.5).)

(12.7) Equality $=$ implies set-theoretical equality $\widehat{=}$. (Proof. By (2.1) and (11.3).)

(12.8) Set-theoretical equality $\widehat{=}$ imples equality $=$ for sets. Namely, $\forall s(s \in x \equiv s \in y) \rightarrow x=y$.

Proof $/ A \rightarrow d$.

A) Assume $x \hat{=} y$ i.e. $\forall s(s \in x \equiv s \in y$ ) by (2.1) and (11.3).

b)) $\quad x \subseteq y \quad / b A \rightarrow b c,(2.15) . \quad$ bA) $\quad \forall T ! T \in x$.

bb) $\quad \$(T) \quad / b A,(2.6),(11.7)$ be) $\quad T \in y \quad / A, b A, b b,(11.2)$.

c) $y \subseteq x \quad /$ similarly to $b . \quad$ d) $x=y \quad / b, c,(2.17)$.

(12.9) Set-theoretical equality $\widehat{=}$ is equivalent to equality $=$ for sets. (Proof. By (12.7) and (12.8).)

(12.10) Set-theoretical equality $\widehat{=}$ is reflexive, symmetric, and transitive. (Proof. By (2.1) and (11.3).)

(12.11) $X \subseteq y \equiv X \subseteq y$. (Any object is a generalized subobject of a set if and only if the object is a subobject of the set. Proof. By (2.15), (2.21), and (12.5). )

(12.12) Inclusion $\subseteq$ implies set-theoretical inclusion $\widehat{\subseteq}$. (Proof. By (2.15) and (11.3). )

(12.13) $x \subseteq \hat{\subseteq} Y x \subseteq Y$. (Proof. By (2.6), (2.15), (11.2), (11.3), and (11.7).)

(12.14) $x \widehat{\subseteq} Y \equiv x \subseteq Y$. (Any set is a set-theoretical subobject of an object 
if and only if the former is a subobject of the latter. Proof. By (12.12) and (12.13). )

(12.15) Set-theoretical inclusion $\hat{\subseteq}$ is reflexive and transitive. (Proof. By (2.15) and (11.3).)

(12.16) $x \hat{\varnothing} Y \equiv x \emptyset Y$. (Proof. By (2.6), (6.1), (11.2), (11.3), and (11.7).)

(12.17) $Z\{x, y\} \rightarrow|Z\{x, y\}|$. Especially, $Z\{=) x \rightarrow|Z\{=) x|$. (Proof. By (6.9), (11.3), and (12.9).)

\section{(13) Set-theoretical satellites and set-theoretical descendents}

In this Section, we describe some properties of the set-theoretical satelliterelation $\widehat{\sigma}$ and the set-theoretical descendent-relation $\widehat{\delta}$. To make our description simpler, we introduce the notation for set-theoretical products of binary relations at first.

(13.1) DF. $X\left(\Gamma^{*} \Delta\right) Y ₹: \exists s \cdot X \Gamma s \Delta Y$. The relation $\Gamma^{*} \Delta$ is called the settheoretical product of the two binary relations $T$ and $\Delta$.

(13.2) $|X \Gamma \Delta Y| \equiv X\left(\hat{\Gamma}^{*} \widehat{\Delta}\right) Y$. (Proof. By (11.3) and (13.1).)

(13.3) The set-theoretical relation-product $\Gamma^{*} \Delta$ of any two binary relations $\Gamma$ and $\Delta$ implies the relation product $\Gamma \Delta$ of them. The set-theoretical relationproduct is monotone in the sense that the relation $\Gamma^{*} \Delta$ implies the relation $\Theta^{*} A$ if $\Gamma$ and $\Delta$ implies $\Theta$ and $A$ respectively. Also, the set-theoretical relationproduct together with the relation product is associative in the sense that the relations $\Gamma(\Delta \Theta), \Gamma^{*}(\Delta \Theta), \Gamma\left(\Delta^{*} \Theta\right)$, and $\Gamma^{*}\left(\Delta^{*} \Theta\right)$ are equivalent to the relations $(\Gamma \Delta) \Theta,\left(\Gamma^{*} \Delta\right) \Theta,(\Gamma \Delta)^{*} \Theta$, and $\left(\Gamma^{*} \Delta\right)^{*} \Theta$, respectively. Accordingly we usually omit the parentheses denoting the order of combinations.

Any relation of the form $\Gamma^{*}(\Delta \wedge \Theta)$ implies $\Gamma^{*} \Delta$ as well as $\Gamma^{*} \Theta$, and any relation of the form $(\Gamma \wedge \Delta)^{*} \Theta$ implies $\Gamma^{*} \Theta$ as well as $\Delta^{*} \Theta$. Also, the settheoretical relation-product is distributive over the combination $V$ in the sense that any relation of the form $\Gamma^{*}(\Delta \vee \Theta)$ is equivalent to $\Gamma^{*} \Delta \vee \Gamma^{*} \Theta$ and any relation of the form $(\Gamma \vee \Delta)^{*} \Theta$ is equivalent to $\Gamma^{*} \Theta \vee \Delta^{*} \Theta$. Further, any relation of the form $\Gamma^{*} \Delta$ is, just as the relation $\Gamma \Delta$ in (2.8), right invariant if $\Delta$ is so, it is left invariant if $\Gamma$ is so, and it is invariant if $\Gamma$ is left invariant and $\Delta$ is right invariant. (Proof. By Definition (13.1).) 
(13.4) $X \Gamma \in y \cdot \equiv \cdot X \Gamma^{*} \in y, X \Gamma=y \cdot \equiv \cdot X \Gamma^{*}=y$, and $x=\Gamma Y \cdot \equiv \cdot x={ }^{*} \Gamma Y$. (Proof. $X \Gamma \in y \rightarrow X \Gamma^{*} \in y$ can be proved by (2.6), (11.2), (11.7), and (13.1). $X \Gamma=y \rightarrow$ $X \Gamma^{*}=y$ and $x=\Gamma Y \rightarrow x={ }^{*} \Gamma Y$ can be proved by (2.3), (11.2), (11.6), and (13.1). $X \Gamma^{*} \in y \rightarrow X \Gamma \in y, X \Gamma^{*}=y \rightarrow X \Gamma=y$, and $x={ }^{*} \Gamma Y \rightarrow x=\Gamma Y$ hold by (13.3).)

(13.5) $X \hat{\in} y \rightarrow X \hat{\in} y$. (Proof. By (1.2), (2.1), (11.3), (12.7), and (13.1).)

(13.6) $x \hat{\in} Y \rightarrow x \in \hat{X}$. (Proof. By (1.2), (2.1), (11.3), (12.8), and (13.1).)

(13.7) Membership $\in$, the generalized membership $€$, and the settheoretically generalized membership $\hat{\in}$ are mutually equivalent for sets. (Proof. By (12.5), (13.5), and (13.6).)

(13.8) $X \subseteq y \rightarrow X \hat{\subseteq} y$.

Proof $/ A \rightarrow b . \quad$ A) $\forall X y ! X \subseteq y$.

b) ) $X \hat{\subseteq} y \quad / b A \rightarrow b d,(1.3),(11.3)$. bA) $\forall s ! s \hat{\in} X$.

bb) $s \Subset X \quad b A,(13.6)$ bc) $s \in y \quad / b b, A,(2.14)$.

bd) $s \hat{\in} y \quad / b c,(13.5)$.

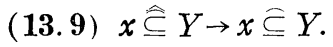

Proof $/ A \rightarrow b . \quad$ A) $\forall x Y ! x \cong ิ$.

b)) $x \subseteq Y \quad / b A \rightarrow b e,(1.3) . \quad$ bA) $\quad \forall S ! S \in x$.

bb) $\quad \$(S) \quad / b A,(11.7) . \quad$ bc) $\quad S \hat{\Theta} x \quad / b A,(13.5)$.

bd) $S \hat{\in} Y / A, b b, b c,(1.3),(11,2)$, (11.3).

be) $S \in \bar{E} \quad / b b, b d,(11.2),(13.6)$.

(13.10) Inclusion $\subseteq$, the generalized inclusion $\subseteq$, the set-theoretical inclusion $\widehat{\subseteq}$, and the set-theoretically generalized inclusion $\widehat{\widehat{C}}$ are mutually equivalent for sets. (Proof. By (12.11), (12.14), (13.8), and (13.9).)

(13.11) $X\{\hat{E}) y \rightarrow|X\{€) y|$. (Proof. By (11.3) and (13.7).)

(13.12) $|x\{\hat{E}) y| \rightarrow x\{\hat{\in}) y . \quad$ (Proof. By (2.6), (11.2), (11.3), (11.7), and (13.7).)

(13.13) $|x\{€) y| \equiv x=y$. (Proof. By (12.6), (13.11), and (13.12).)

(13.14) $\sigma(p) \rightarrow \widehat{\sigma}(p)$. (Proof. By (1.4), (11.3), (11.7), and (13.10). )

(13.15) The set-theoretical satellite-relation $\widehat{\sigma}$ implies the satellite relation $\sigma$ for sets. 


\section{Proof $/ A \rightarrow c . \quad$ A) $\forall x y ! x \widehat{\sigma} y$.}

b) $\forall p\left(\widehat{\sigma}(p) \wedge y \in^{*} \in p \cdot \rightarrow x \in \in^{*} \in p\right) \quad / A,(1.5),(11.3)$, (13.2).

c)) $\quad x \sigma y \quad / c A \rightarrow c r$. cA) Define $\Sigma$ by $X \Sigma Y ₹ \$(X)$.

cb) $\exists P ! P\{=\Sigma \wedge \sigma) y /(4.2)$.

cc) $\forall T(T \in P \rightarrow \$(T)) \quad / c c A \rightarrow c c d$. ccA) $\forall T ! \quad T \in P$.

ccb) $\exists S ! T=S \Sigma y \quad / c c A, c b . \quad$ cce) $\$(S) \quad / c c b, c A$.

ced) $\quad \$(T) / c c c,(11.6),(11.2)$.

cd) $P\{\hat{\in}) P \quad / c b,(2.3),(2.8),(2.9),(3.5),(2.29)$.

ce) $\$(P) / c c, c d,(11.15) . \quad$ cf) $\exists p ! p\{=\Sigma \wedge \sigma) y \quad / c b, c e,(11.2)$.

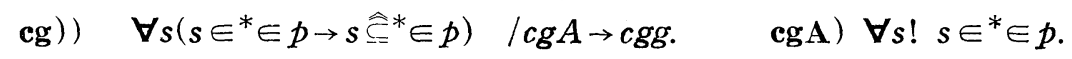

cgb) $\exists t ! s \in t \in p \quad \operatorname{lcg} A,(13.1)$ cgc) toy $/ c g b, c f$.

cgd) $\quad s \sigma y$ lcgb, cgc, (3.1), (3.2). cge) $s=s \Sigma y \quad /(2.3),(11.4), c A$.

cgf) $s \stackrel{\hat{\subseteq}}{\subseteq} \in p \quad /(13.10),(2.13), c g d, c g e, c f$.

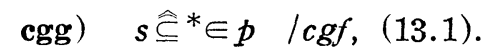

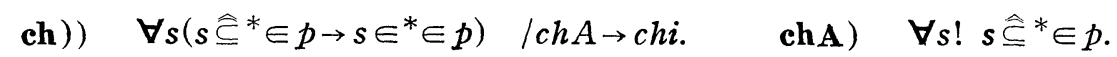

chb) $\exists t ! s \subseteq t \in p \quad / \operatorname{ch} A,(13.1),(13.10)$.

che) $\exists u ! u\{=) s /(5.4),(11.8),(11.2)$. chd) toy /chb, cf.

che) $u \sigma y$ /chc, chb, chd, (3.1), (3.3), (3.4).

chf) $u=u \Sigma y \quad /(2.3),(11.4), c A . \quad$ chg) $u \in p \quad / c h e, c h f, c f$.

chh) $s \in u$ /chc, (2.26). chi) $s \in *^{*} \in p$ /chh, chg, (13.1).

ci) $\widehat{\sigma}(p) \quad / c g, c h .(1.4),(11.3),(13.2)$.

cj) $\exists z ! z\{=) y \quad /(5.4),(11.8),(11.2)$. ck) $y \in z \quad / c j,(2.26)$.

cl) $z=z \Sigma y \quad /(2.3),(11.4), c A$. cm) $z \sigma y \quad / c j,(3.4)$.

cn) $z \in p \quad / c l, c m, c f . \quad$ co) $y \in *^{*} \in p \quad / c k, c n,(13.1)$.

ep) $\exists w ! x \in w \in p \quad / c i, c o, b,(13.1)$. eq) woy /cp, cf.

cr) $\quad x \sigma y \quad / c p, c q,(3.1),(3.2)$.

(13.16) $|\forall S(S \in \in p \rightarrow S \in p)| \equiv \forall S(S \in \in p \rightarrow S \in p)$ (Proof. By (2.6), (11.2), (11.3), (11.7), (13.1), (13.2), and (13.3).)

(13.17) $X \delta y \rightarrow X \widehat{\delta} y$. (Proof. By (7.1), (11.3), (13.5), (13.6), and (13.16).) (13.18) $x \widehat{\delta} y \rightarrow x \delta y$.

Proof $/ A \rightarrow l . \quad$ A) $\forall x y ! x \widehat{\delta} y$.

b) $\forall p\left(\forall s\left(s \in \in^{*} \in p \rightarrow s \in p\right) \rightarrow(y \in p \rightarrow x \in p)\right) \quad / A,(7.1),(11.3),(13.2)$, (13.7). 

c) $\exists P ! P\{=\delta \wedge \sigma) y \quad /(4.2)$.
d) $P\{\delta) y \quad / c,(7.6),(7.5),(2.2)$.
e) $\quad \forall T(T \in P \rightarrow \$(T)) \quad / e A \rightarrow e c$.
eA) $\forall T ! T \in P$.
eb) $T \delta y \quad / e A, d$.
ec) $\quad \$(T) \quad / e b,(11.12)$.
f) $P\{\in \in) P \quad / d,(2.29),(7.6)$.
g) $\$(P) \quad / e, f,(11.15)$.
h) $\exists p ! p\{\delta) y / d, g,(11.2)$.
i)) $\forall s\left(s \in \in^{*} \in p \rightarrow s \in p\right) \quad / i A \rightarrow i e$.
iA) $\forall s ! s \in *^{*} \in p$.
ib) $\exists t ! s \in t \in p \quad / i A,(13.1)$.
ic) $t \delta y \quad / i b, h$.
id) $s \delta y \quad / i b, i c,(2.6),(7.3),(7.2)$.
ie) $s \in p \quad / i d, h$.
j) $y \in p \quad / h,(7.2)$.
k) $x \in p \quad / i, j, b . \quad$ l) $x \delta y \quad / k, h$.

(13.19) The descendent relation $\delta$ and the set-theoretical descendent-relation $\hat{\delta}$ are equivalent for sets. (Proof. By (13.17) and (13.18).)

\section{(14) Set-theoretical images of axioms and theorems}

In this Section, we prove that the set-theoretical images of axioms and theorems (without capital-letter free-parameters) are provable in OF. (See (14.3) and (14.4). Compare $O Z$-(6.1).) By virtue of this proof, we can see also that $\mathbf{O Z}$ can be imbedded in OF. (See (14.6).)

To prove the above meta-theorem, we make use of a theorem, which can be regarded as the set-theoretical image of a presumably unprovable proposition in $\mathbf{O F}$. (See (14.1).) By virtue of this theorem, we can also introduce term symbols in our theory of sets. (See (15.1).) In addition to this theorem, there are some important theorems of this kind, a couple of which are described in this Section.

(14.1) $x \widehat{=} y \rightarrow|\mathfrak{T}(x) \equiv \mathfrak{A}(y)|$, where any number of free set-variables may occur in $\mathfrak{A}(t)$. (We can prove this meta-theorem by making use of (2.1), (11.3), (12.4), and (12.9).)

(14.2) Any relation of the forms $=\hat{\Gamma}, \hat{\Gamma}=$, and $=\hat{T}=$ as well as any relation of the forms $\widehat{=}{ }^{*} \widehat{\Gamma}, \hat{\Gamma}^{*} \hat{=}$, and $\widehat{=}{ }^{*} \hat{\Gamma}^{*} \hat{=}$ is equivalent to the relation $\hat{\Gamma}$ for sets. In other words, any relation of the form $\hat{\Gamma}$ is set-theoretically invariant. Here, any number of free set variables may occur in $\hat{T}$. (Proof. By (12.8), (13.1), and (14.1).) 
(14.3) $\left|\exists P \cdot P\left\{\langle\Gamma)_{\sigma}\right) m\right|$, where $P$ should not occur in $\Gamma$ but any number of free set-variables may occur in $\Gamma$. (The set-theoretical image of any axiom of $\mathrm{OF}$ is provable in $\mathrm{OF}$. Compare $O Z-(6.1 .1)$.)

Proof $/ A \rightarrow k . \quad$ A) Define $\Delta$ by $X \Delta Y * \cdot X={ }^{*} \hat{\Gamma}^{*}=Y \hat{\sigma} m$.

b) $\exists P ! P\left\{\{\Delta)_{\sigma}\right) m$ /axiom.

c)) $\forall T(T \in P \rightarrow \$(T)) \quad / c A \rightarrow c e . \quad$ cA) $\forall T$ ! $T \in P$.

cb) $\exists U ! T\{\Delta) U_{\sigma m} / c A, b$.

cc) $\quad \forall W(W \in T \rightarrow \$(W)) \quad / c c A \rightarrow c c c$.

ccA) $\forall W ! W \in T$. ccb) $W \Delta U \quad / c c A, c b$.

ccc) $\quad \$(W) \quad / c c b, A,(11.6),(13.1)$.

cd) $\quad T\{\hat{\in}) T \quad / c b, A,(13.3),(2.3),(2.29)$. $\quad$ ce) $\quad \$(T) \quad / c c, c d,(11.15)$.

d) $P\{\in) P \quad / b,(2.8),(2.10),(2.29)$. e) $\$(P) \quad / c, d,(11.15)$.

f) $\exists p ! p\left\{\{\Delta)_{\sigma}\right) m \quad / b, e,(11.2)$.

g)) $\forall t\left(t \in p \rightarrow \cdot\left|t\{\Gamma)_{\sigma m}\right| \vee \varnothing(t)\right) \quad / g A \rightarrow g k . \quad$ gA) $\forall t ! t \in p \wedge>\emptyset(t)$.

gb) $t\{\Delta)_{\sigma m} \lg A, f . \quad$ ge) $\exists Y ! t\{\Delta) Y \sigma m \quad / g b$.

gd) $\exists S ! S \in t \quad / g A,(6.1)$ ge) $S \Delta Y \quad / g d, g c$.

gf) $S={ }^{*} \hat{\Gamma}^{*}=Y \hat{\sigma} m$ lge, $A$.

gg) $\quad S(Y) \quad \lg f,(2.3),(11.6),(13.1)$.

gh) ) $\forall s(s \in t \rightarrow s \hat{\Gamma} Y) \quad$ /ghA $\rightarrow$ ghd.

ghA) $\forall s ! s \in t . \quad$ ghb) $s \Delta Y \quad / g h A, g c$.

ghc) $s={ }^{*} \hat{\Gamma}^{*}=Y \quad$ lghb, A. $\quad$ ghd) $s \hat{\Gamma} Y \quad$ lghc, gg, (14.2).

gi) ) $\forall s(s \hat{\Gamma} Y \rightarrow s \in t) \quad$ /giA $\rightarrow$ gic. giA) $\forall s ! s \hat{\Gamma} Y$.

gib) $s \Delta Y \quad / A, g i A, g f, g g,(2.3),(11.2),(13.1)$. gic) $s \in t \quad / g i b, g c$.

gj) $|t\{\Gamma) Y|$ lgh, gi, (11.3). gk) $\left|t\{\Gamma)_{o m}\right|$ lgj, gg, gf, (11.2).

h) ) $\forall t\left(\left|t\{\Gamma)_{\sigma m}\right| \rightarrow t \in p\right) \quad / h A \rightarrow h g . \quad$ hA $) \quad \forall t !\left|t\{\Gamma)_{\sigma m}\right|$.

hb) $\exists y !|t\{\Gamma) y| \wedge y \widehat{\sigma} m \quad / h A,(13.2)$, (13.1).

he) $\quad \forall S(S \in t \rightarrow S \Delta y) \quad / h c A \rightarrow h c d . \quad$ heA) $\quad \forall S ! S \in t$.

hcb) $\quad \$(S) \quad / h c A,(2.6),(11.7) . \quad$ hec) $S \hat{\Gamma} y / h c A, h c b, h b,(11.2),(11.3)$.

hed) $S \Delta y / h c c, h c b, h b, A,(2.3),(11.2),(13.1)$.

hd) $\quad \forall S(S \Delta y \rightarrow S \in t) \quad / h d A \rightarrow h d e$.

hdA) $\forall S ! S \Delta y . \quad$ hdb) $\quad S={ }^{*} \widehat{\Gamma}^{*}=y \quad / h d A, A$.

hde) $\$(S) \quad / h d b,(11.6),(13.1) . \quad$ hdd) $S \widehat{\Gamma} y / h d b, h d c,(14.2)$.

hde) $S \in t \quad / h d c, h d d, h b,(11.2),(11.3)$.

he) $t\{\Delta\}^{\prime} / h c, h d$. hf) $t\{\Delta)_{\sigma m} / h e, h b,(13.15)$. 
hg) $t \in p \quad / h f, f$.

i)) $\exists n\left(\varnothing(n) \wedge\left|n\{\Gamma)_{\sigma} m\right|\right) \rightarrow\left|p\left\{\{\Gamma)_{\sigma}\right) m\right| \quad / i A \rightarrow i b$.

iA) Assume $\exists n ! \varnothing(n) \wedge \mid n\{\Gamma)_{\sigma m !}$

ib)) $\left|p\left\{\{\Gamma)_{\sigma}\right) m\right| \quad / i b a, h$.

iba)) $\forall t\left(t \in p \rightarrow \mid t\{\Gamma)_{\sigma m}\right) \quad / i b a A \rightarrow i b a d . \quad$ ibaA $\quad \forall t ! t \in p$.

ibab) $\left|t\{\Gamma)_{\sigma m}\right| \vee \emptyset(t) \quad / i b a A, g$.

ibac)) $\quad \varnothing(t) \rightarrow\left|t\{\Gamma)_{\sigma m}\right| \quad / i b a c A \rightarrow$ ibacc. ibacA) Assume $\varnothing(t)$.

ibacb) $\quad t=n \quad \mid i b a c A, i A,(6.8) . \quad$ ibacc) $\quad|t\{\Gamma) \circ m| \quad \mid i b a c b, i A,(14.1)$.

ibad) $\left|t\{\Gamma)_{\sigma m}\right| \quad / i b a b, i b a c$.

j) ) > $\exists n\left(\varnothing(n) \wedge\left|n\{\Gamma)_{\sigma} m\right|\right) \rightarrow \exists q \mid q\left\{\langle\Gamma)_{\sigma}|m| \quad \mid j A \rightarrow j b\right.$.

jA) Assume $>\exists n\left(\varnothing(n) \wedge \mid n\{\Gamma)_{\sigma m}\right)$.

jb) ) $\exists q\left|q\left\{\{\Gamma)_{\sigma}\right) m\right| \quad / j b A \rightarrow j b i$. jbA) Define $\Theta$ by $X \Theta Y$ 과 $\neg(X)$.

jbb) $\exists Q ! Q\{=(\Theta \wedge \in)) p \quad /(4.4)$.

jbc) $Q\{\in) Q \quad / j b b,(2.3),(2.8),(2.29)$.

jbd) $\quad \forall T(T \in Q \rightarrow \$(T)) \quad(j b d A \rightarrow j b d d . \quad$ jbdA $) \quad \forall T ! T \in Q$.

jbdb) $\quad \exists W ! T=W(\Theta \wedge \in) p \quad / j b d A, j b b$.

jbdc) $\quad \$(W) \quad / j b d b,(2.6),(11.7)$.

jbdd) $\quad \$(T) / j b d b, j b d c,(11.2),(11.6)$.

jbe) $\quad \$(Q) \quad / j b c, j b d,(11.15)$.

jbf) $\exists q ! q\{=(\Theta \wedge \in)) p \quad / j b b, j b e,(11.2)$.

jbg) $) \quad \forall t(t \in q \rightarrow \cdot t \in p \wedge>\varnothing(t)) \quad / j b g A \rightarrow j b g c, j b g f$.

$\operatorname{jbg} \mathbf{A}) \quad \forall t ! t \in q . \quad \mathbf{j b g b}) \quad t=(\Theta \wedge \in) p \quad \operatorname{jbg} A, j b f$.

jbgc $\quad t \in p \quad / j b g b,(12.1) . \quad$ jbgd $) \quad \exists W ! t=W \Theta p \quad / j b g b$.

jbge $) \quad \neg \varnothing(W) \quad / j b g d, j b A . \quad$ jbgf $) \quad \neg \emptyset(t) \quad$ libge, jbgd, (6.7).

jbh) ) $\forall t(t \in p \wedge \neg \emptyset(t) \cdot \rightarrow t \in q) \quad / j b h A \rightarrow j b h d$.

jbhA ) $\forall t ! t \in p \wedge>\varnothing(t)$.

jbhb) $\quad t \Theta p \quad / j b h A, j b A . \quad$ jbhc $) \quad t=(\Theta \wedge \in) p \quad / j b h b, j b h A,(2.3)$.

jbhd) $\quad t \in q \quad / j b h c, j b f$.

jbi) ) $\left|q\left\{\langle\Gamma)_{\sigma}\right) m\right| \quad / j b i a, j b i b,(11.2),(11.3)$.

jbia)) $\forall t\left(t \in q \rightarrow\left|t\{\Gamma)_{\sigma m}\right|\right) \quad(j b i a A \rightarrow j b i a c . \quad$ jbiaA $) \quad \forall t ! t \in q$.

jbiab) $\quad t \in p \wedge \neg \varnothing(t) \quad / j b i a A, j b g$. jbiac) $\left|t\{\Gamma)_{\sigma m}\right| \quad \mid j b i a b, g$.

jbib)) $\forall t\left(\left|t\{\Gamma)_{\sigma m}\right| \rightarrow t \in q\right) \quad / j b i b A \rightarrow j b i b a$ jbibA $\quad \forall t !\left|t\{\Gamma)_{\sigma m}\right|$.

jbibb) $\quad t \in p \quad / j b i b A, h . \quad$ jbibc $) \quad \neg \varnothing(t) \quad / j b i b A, j A$.

jbibd) $\quad t \in q \quad / j b i b b, j b i b c, j b h$. 
k) $\exists q\left|g\left\{\{\Gamma)_{\sigma}\right) m\right| \quad \mid i, j$.

(14.4) Remark. (14.3) implies that the set-theoretical image of every theorem of OF is provable in OF. (Compare OZ-(6.1.2).) Of course, we regard hereby that all the parameters seemingly free in the theorem as bound variables which are bound to the removed universal quantifiers standing at the top of the theorem.

With respect to free set parameters, we can now talk of the set-theoretical image of a proposition without assigning its special expression, because the set-theoretical images of any two equivalent propositions are equivalent to each other. (Compare OZ-(6.1.4).)

(14.5) By (14.4), we can regard the set-theoretical image of every theorem as a new theorem. In the following, we refer to the set-theoretical image of a theorem $(a, b)$ by $(a, b)^{*}$.

It should be remarked here that we must be careful when we make the set-theoretical image of a theorem seemingly containing free set-variables. For example, the first formula of $(11.4)^{*}$ is not the formula $|\$(x)|$, which means $\forall X(\$(X) \rightarrow|\$(X)|)$, but it means $\forall x(|\$(x)| \rightarrow|\$(x)|)$ since the first formula $S(x)$ of (11.4) is an abbreviation of $\forall X(S(X) \rightarrow S(X)$ ). (See (11.3), (14.4), (11.4), and also (14.8)-(14.10).)

(14.6) We can imbed $\mathbf{O Z}$ in $\mathbf{O F}$ by the interpretation that we regard proto-membership " $\epsilon$ " of $\mathbf{O Z}$ as membership " $\in$ " of $\mathbf{O F}$ and the field of objects $X$ of $\mathrm{OZ}$ as the field of sets $x$ of $\mathrm{OF}$, accordingly, we regard the satellite relation " $\sigma$ " of $\mathrm{OZ}$ as the set-theoretical satellite relation " $\widehat{\sigma}$ " of $\mathrm{OF}$. (Proof. By OZ-(1.1.2), OZ-(1.1.3), (1.4), (1.5), (2.15), (11.3), (13.1), and (13.10). See foot-note 9).) Accordingly any axiom of $\mathbf{O Z}$ is interpreted as a proposition of the form $\forall u \cdots w m \exists p \forall x(x \in p \equiv \cdot \widehat{\mathfrak{l}}(x) \wedge x \widehat{\sigma} m)$ which contains no more free variables.

Now, let $\mathfrak{H}(X)$ be the formula obtained by replacing all the variables in $\widehat{\mathfrak{I}}(x)$ other than $u, \ldots, w$, and $m$ by their corresponding object variables, and let $\Gamma$ be the binary relation defied by $X \Gamma Y ₹ \mathfrak{H}(X)$, where $Y$ is a variable which does not occur in $\mathfrak{H}(X)$. Then, evidently holds $\forall x y(x \widehat{\Gamma} y \equiv \widehat{\mathfrak{I}}(x))$ in OF.

By $(4.2)^{*}, \forall u \cdots w m \exists p !|p\{=\Gamma \wedge \sigma) m|$. For this $p$, we can further prove $\forall x(x \in p \equiv \cdot \widehat{\mathfrak{H}}(x) \wedge x \widehat{\sigma} m)$ by making use of (11.3), (13.1), and (14.2). Thus 
$\mathbf{O Z}$ can be imbedded in $\mathbf{O F}$.

(14.7) $|x\{\hat{E}) x|$ (Any set is set-theoretically normal. Proof. By (11.4), (9.2), and (13.11).)

(14.8) $\hat{\nu}(x)$. (Any set is set-theoretically totally-normal. Proof. By (14.7), (9.1), and (11.3).)

(14.9) $\hat{\rho}(x)$. (Any set is set-theoretically regular.)

$$
\text { Proof } / A \rightarrow h \text { (reductio ad absurdum). A) Assume } \rightarrow \widehat{\rho}(x) \text {. }
$$

b) $x \hat{\rho}^{*} \ni^{*} \widehat{\delta} x \quad / A,(8.1),(11.3),(13.2)$.

c) $\exists y$ ! x $\hat{\rho} y \ni^{*} \hat{\delta} x \quad / b,(13.1)$.

d) $y \ni \delta x / c,(13.1),(13.18)$.

e) $\forall t\left(t \widehat{\delta} x \wedge t \in y \cdot \rightarrow t \ni^{*} \in y\right) \quad / c,(8.1),(11.3),(13.2)$.

f)) $\quad x \rho y \quad / f A \rightarrow f d,(8.1)$ fA) $\forall T ! T \delta x \wedge T \in y$.

fb) $\quad S(T) / f A,(2.6),(11.7), \quad$ fe) $\quad T \hat{\delta} x \quad / f A,(13.17)$.

fd) $T \ni \in y \quad / f A, f b, f c, e,(11.2),(13.3)$.

g) $>\rho(x) / f, d,(8.1) . \quad$ h) contradiction $/ g,(11.4)$.

(14.10) $\widehat{\$}(x)$. (Any set is a set-theoretical set. Proof. By (11.1), (11.3), (14.8), and (14.9).)

\section{(15) A theory of sets}

In this Section, we show that the Fraenkel set-theory SF (without the axiom of choice) can be imbedded in OF. To prove this, we show that all the axioms of SF hold in the field of sets in OF. In the proofs of these interpreted axioms, we make use of (11.2), (11.3), and (13.1) without notice. For convenience' sake, we introduce term symbols in our theory of sets, although they are not indispensable for our system.

(15.1) Term symbols. If there is a set $p$ satisfying $\forall s(s \in p \equiv \mathfrak{A}(s))$, then $\forall s(s \in q \equiv \mathfrak{U}(s))$ implies $\boldsymbol{q} \widehat{=} p$; so, according to (14.1), we do not have to draw any distinction between these sets $p$ and $q$ in our set theory, since $\hat{=}$ can be regarded as equality. We denote the set $p$ by the term symbol $\{s ; \mathfrak{U}(s)\}$ as in $\mathbf{O Z}^{\prime}$ or in other ordinary set-theories. (See $O Z$-(6.3). Concerning the meaning of the set-theoretical images of propositions, in which term symbols occur, see $O Z-(6.3 .4)$.) For any term symbol $t$ holds evidently $\$(t)$. In the 
following we make use of this fact without notice.

(15.2) Unit sets and pair sets. By $(6.9)^{*}$ and $(6.11)^{*}$, we can introduce the term-symbol $\{x, y\}$ (pair set of $x$ and $y$ ) which denotes $\{s ; s \hat{=} y \vee s \hat{=} y\}$. $\{x, x\}$ is denoted by $\{x\}$ (unit set of $x$ ) as usual. By (6.9)*, hold $s \in\{x, y\}$ $\equiv \cdot s \hat{=} x \wedge s \hat{=} y \quad$ and $\quad s \in\{x\} \equiv s \hat{=} x . \quad$ (Compare $O Z$-(6.5).)

(15.3) Sum sets. By $(5.3)^{*}$, we can introduce the term symbol $\Subset(m)$ (sum set of $m$ ) which denotes $\left\{s ; s \hat{\in}^{*} \in m\right\}$. By (13.7) holds $s \in €(m) \equiv s \in{ }^{*} \in m$. (Compare OZ-(6.7.10).)

(15.4) Power sets. By $(5.2)^{*}$, we can introduce the term symbol $\mathfrak{P}(m)$ (power set of $m$ ) which denotes $\{s ; s \hat{\subseteq} m\}$. Evidently, holds $s \in \mathfrak{P}(m) \equiv s \subseteq \underline{\widehat{C}} m$. (Comdare $O Z$-(6.7.8).)

(15.5) Null set. We can introduce the term symbol $\varnothing$ (null set) which denotes $\{x ; x \hat{\neq} \boldsymbol{x}\}$. We can show by $(2.3)^{*},(6.1)^{*}$, and $(6.5)^{*}$, that $\{\boldsymbol{x} ; \boldsymbol{x \neq} \hat{\neq}\}$ is an admissible term symbol. Evidently holds $\forall s \cdot s \notin 0$. (Compare OZ-(6.4).)

(15.6) Infinite sets. By $(10.15)^{*}$, we can introduce the term symbol (set of all the natural numbers) which denotes $\{s ; \hat{\mathbb{E}}(s)\}$. By (15.2), (15.5), $(6.1)^{*},(10.5)^{*}$, and $(10.6)^{*}$, we can prove easily

$$
\emptyset \in \Psi, \quad \text { and } \quad x \in \# \rightarrow\{x\} \in \Psi \text {. }
$$

These formulas show that the axiom of infinity holds in our set theory. (Compare OZ-(6.9).)

(15.7) Equality. Concerning equality in our theory of sets, hold

$$
x \widehat{=x}, \quad x \widehat{=} y \rightarrow \forall p(x \in p \equiv y \in p), \quad \text { and } \quad \forall s(s \in x \equiv s \in y) \rightarrow x \widehat{=y}
$$

by $(2.1)^{*},(12.10),(12.9)$, and (12.4). The third formula can be regarded as the axiom of extensionality. (Compare OZ-(6.2).)

(15.8) Aussonderung. By $(4.4)^{*}$, we can introduce the term symbol $\{s ;|s=(\Gamma \wedge \in) m|\}$. If we define $\Gamma$ by $X \Gamma Y \mp \mathfrak{A}(X)$, we can see by (14.1) and (12.9) that the term symbol can be also expressed as $\{s ;|\mathfrak{A}(s)| \wedge s \in m\}$. Accordingly, the aussonderung axiom holds in our set theory, because any set-theoretical condition on $s$ can be expressed in the form of $|\mathfrak{T}(s)|$. (Compare $O Z$-(6.7.4).) 
(15.9) Replacement. Let $x \widehat{\Delta} z$ be a relation satisfying $\forall x y(x \widehat{\Delta} z \wedge y \widehat{\Delta} z$. $\rightarrow x \hat{=} y)$. By $(4.5)^{*}$ we can introduce the term symbol $\left.\{s ; \mid s\{\in \Delta) \in m\}\right\}$. We prove now that $|s \Delta \in m|$ implies $|s\{\in \Delta) \in m|$. Namely.

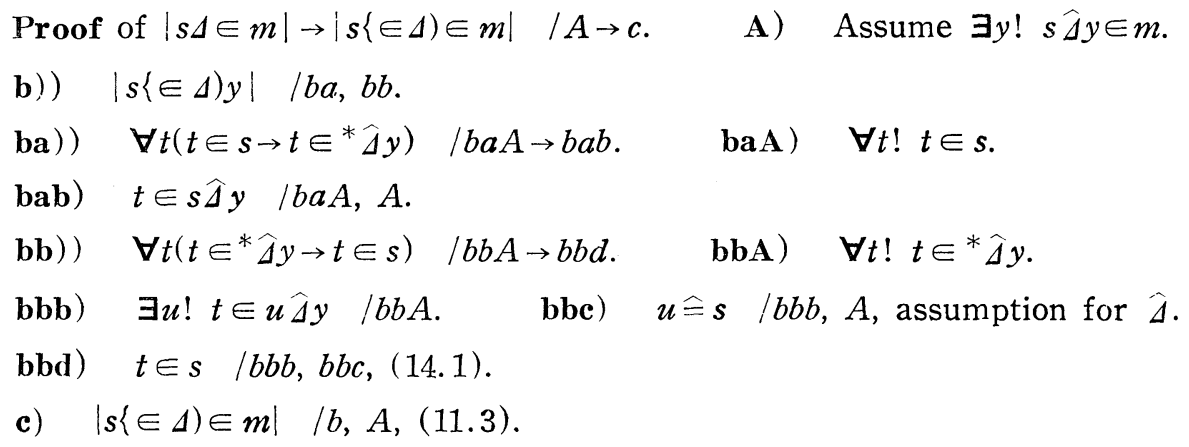

Now by (15.8), we can introduce the term symbol $\{t ;|t \Delta \in m| \wedge t \in\{s ;$ $|s\{\in \Delta) \in m|\}\}$, which can be also expressed as $\{t ;|t \Delta \in m|\}$ by virtue of the above proof.

Thus the axiom of replacement holds in our set theory, because every set-theoretical relation can be expressed in the form of $\widehat{\Delta}$.

(15.10) Fundierung. By reductio ad absurdum, we can prove in our set theory that any non-vacant set $x$ has a member which is disjoint with $x$. Namely,

$$
\text { Proof of } \forall x\left(x \in m \rightarrow x \ni^{*} \in m\right) \rightarrow m \widehat{=\varnothing} \quad / A \rightarrow g \text {. }
$$
A) Assume $\forall x\left(x \in m \rightarrow x \ni^{*} \in m\right) \wedge m \hat{\neq} \emptyset$.
b) $\exists y ! y \in m / A,(2.1)^{*},(15.5)$.
c) $m \hat{\rho} m / A,(8.1)^{*}$.
d) $y \hat{\delta} m / b,(7.3)^{*},(13.7)$.
e) $\quad \boldsymbol{m}^{*} \ni^{*} \widehat{\delta} m \quad / c, b, d$.
f) $\quad>\hat{\rho}(m) / e,(8.1)^{*}$.
g) contradiction $/ f$, (14.8).

$(15.11)$ By (15.2)-(15.10), we see that SF (the Fraenkel set-theory without the axiom of choice) can be imbedded in OF.

Naturally, we can also introduce term symbols such as $x \cup y, x \cap y,\langle x, y\rangle$, $x \times y$, etc., or we can construct a theory of ordinal numbers with recursive functions or recursively defined relations. In this work, however, we do not discuss the matter in detail. (Compare $O Z$-(6.9.15). ) 


\section{References}

[1] K. Ono, A theory of mathematical objects as a prototype of set theory, Nagoya Math. Jour., vol. 20 (1962), pp. 105-168.

[2] K, Ono, On a practical way of describing formal deductions, Nagoya Math. Jour., vol. 21 (1962), pp. 115-121.

[3] E. Zermelo, Untersuchungen über die Grundlagen der Mengenlehre, I, Math. Ann., vol. 65 (1908), pp. 261-281.

[4] A. Fraenkel, Einlitung in die Mengenlehre, 1928 (3. Aufl.)

[5] K. Gödel, The consistency of the axiom of choice and of the generalized continuum hypothesis with the axioms of set theory, Annals of Mathematics Studies, No. 3, 1940.

[6] G. Gentzen, Untersuchungen über das logischen Schliessen, Math. Z., 39, (1935), pp. 176-210, 405-431.

[7] R. O. Gandy, On the axiom of extensionality, Part I, II; J. Symbolic Logic; I. vol. 21 (1956), pp. 36-48; II. vol. 24 (1959), pp. 287-300.

Mathematical Institute

Nagoya University 